\title{
Variations in Rotational Barriers of Allyl and Benzyl Cations, Anions, and Radicals
}

Zhe Li, ${ }^{\mathrm{a}}$ Thomas Bally, ${ }^{* \mathrm{~b}}$ K. N. Houk ${ }^{* \mathrm{a}}$ and Weston Thatcher Borden*c

\section{Supporting Information}

Scheme S1: Experimental thermochemical data for the allyl species $\quad$ S2

Scheme S2: Experimental thermochemical data for the benzyl species $\quad$ S3

Figure S1: Molecular orbitals for the charged benzyl species $\quad$ S4

Figure S2: Molecular orbitals for the benzyl radical S5

Results of PPP-calculations on the allyl cation, anion and radical S6

Geometries of all species calculated and discussed in the paper $\quad$ S10 


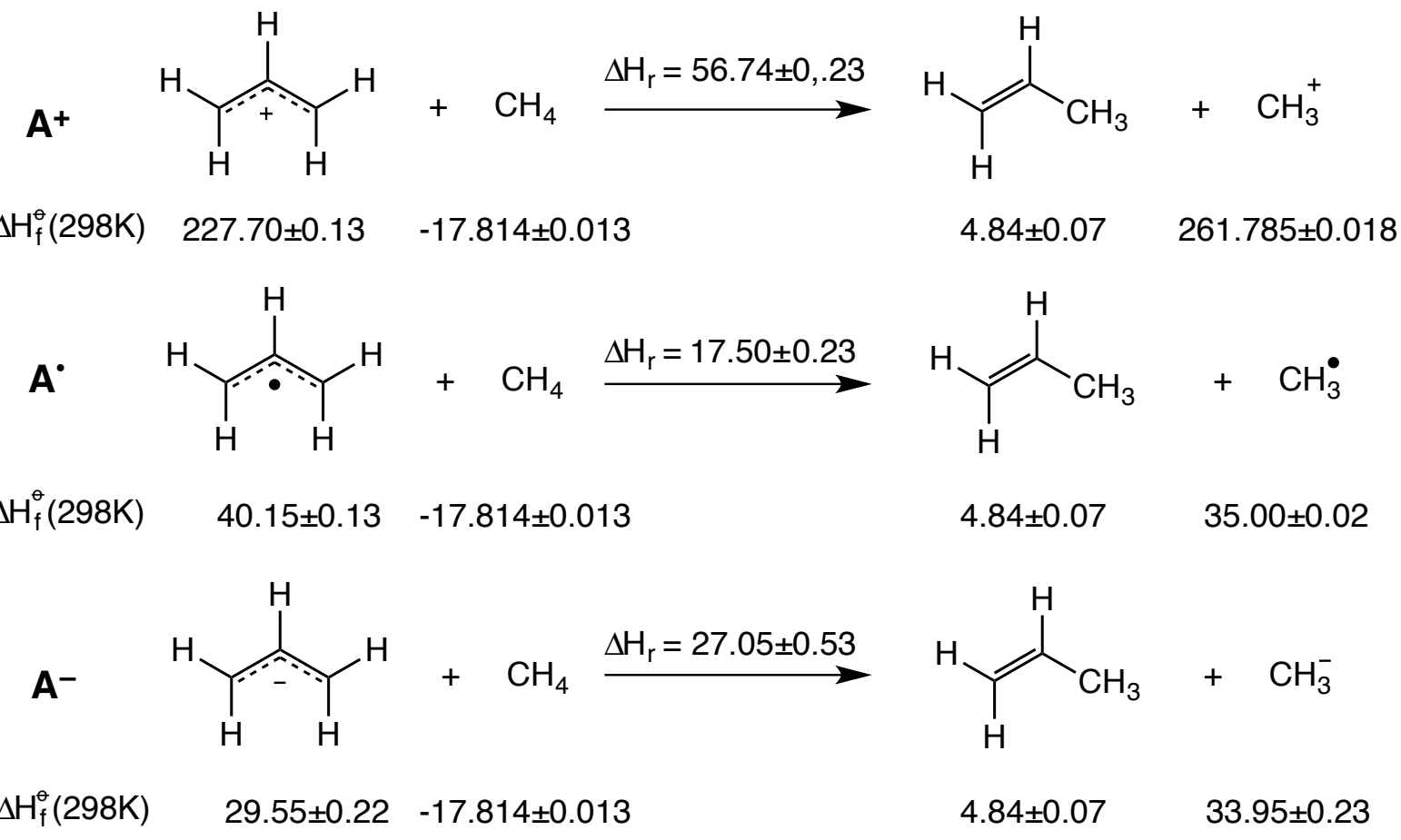

Scheme S1: Thermochemical data for the allyl species from the Active Thermochemical Tables (Ref. 14 in the paper) 
$\mathrm{B}^{+}$<smiles>Cc1ccccc1</smiles>

$\Delta H_{\mathrm{f}}^{\ominus}(298 \mathrm{~K})$

$216.5 \pm 1.1 \quad-17.8 \pm 0.07$

$12.0 \pm 0.26$

$262.0 \pm 0.3$

$B^{\circ}$
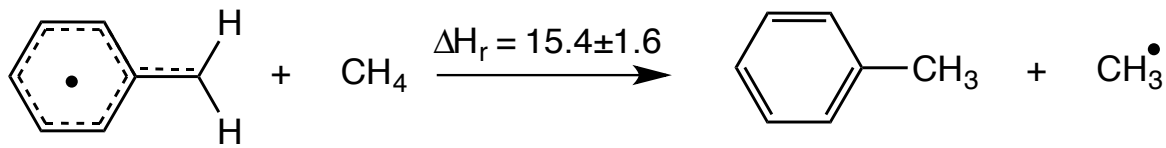

$\Delta H_{\mathrm{f}}^{\ominus}(298 \mathrm{~K})$

$$
49.5 \pm 1.0
$$

$-17.8 \pm 0.07$

$12.0 \pm 0.26$

$35.1 \pm 0.2$

B-

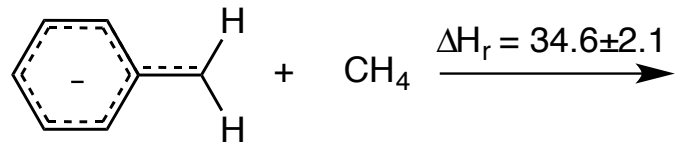

$\Delta H_{\mathrm{f}}^{\ominus}(298 \mathrm{~K})$

$28.5 \pm 1.1$

$-17.8 \pm 0.07$

$12.0 \pm 0.26$

$33.3 \pm 1.0$

Scheme S2: Thermochemical data for the benzyl species. All the data were taken from the NIST Webbook (Ref 15 in the paper) 


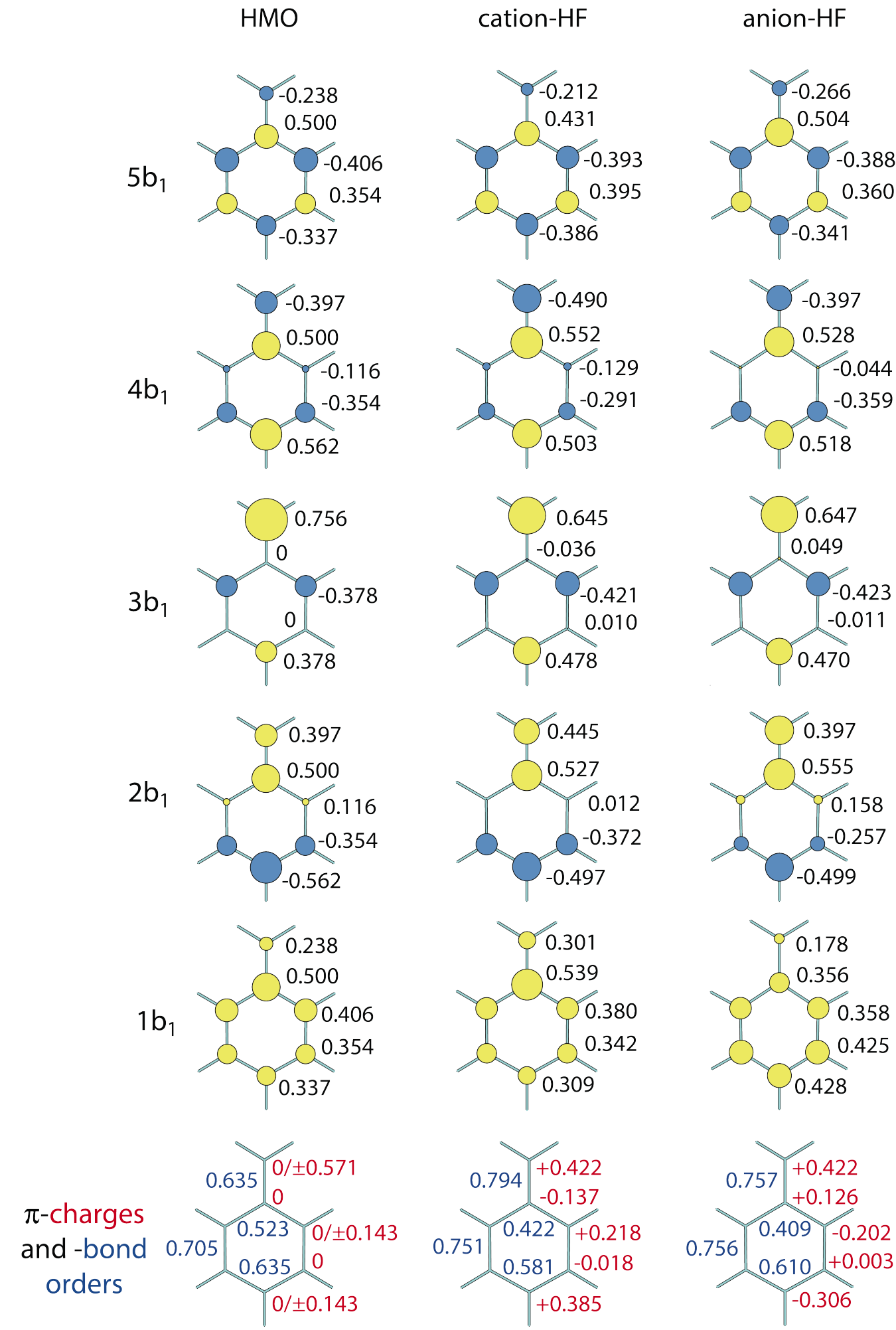

Figure S1: b1- $\pi$-molecular orbitals (with coefficients) obtained from calculations at the HMO level (left column), and at the HF/STO-3G level for the benzyl cation (central column) and the benzyl anion (right column). The HF-MOs were normalized to 1 . At the bottom the $\pi$-charges and -bond orders are listed which were calculated according to HMO theory for the different set of MOs. The MOs shown on the right in Figures 5 and 6 are drawn a bit exaggerated, compared to the "true" HF MOs, to better illustrate the effects of the mixing. 

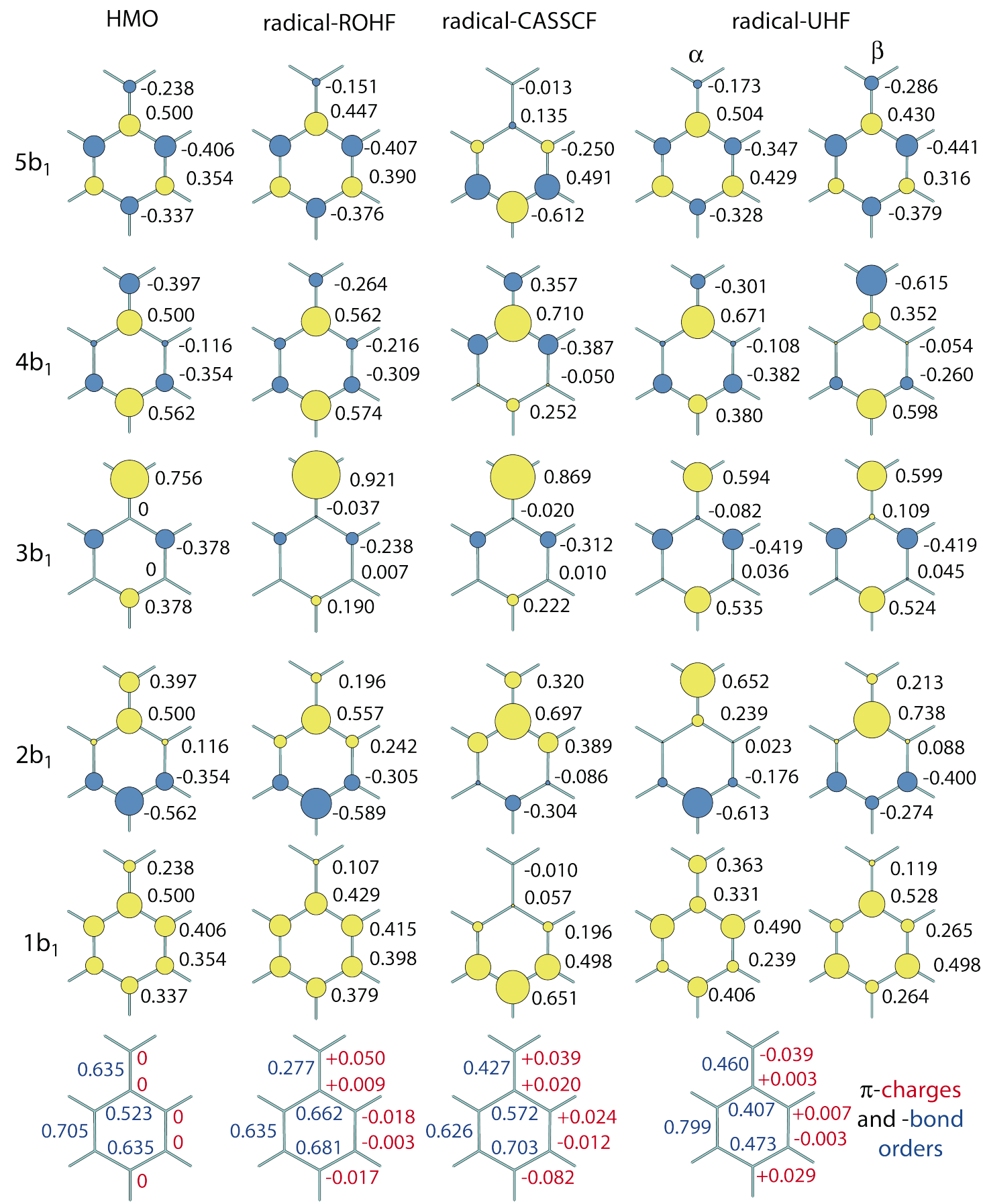

Figure S1: $b_{1}-\pi$-molecular orbitals (with coefficients) obtained from calculations at the HMO level (leftmost column), and, for the radical at three different levels. At the bottom the $\pi$-charges and -bond orders are listed which were calculated according to HMO theory for the different set of MOs. The transfer of spin from the ring to the exocyclic carbon is very well illustrated by the change of the shape of the $3 b_{1}$ MO between HMO and ROHF which results from mixing of the $3 b_{1}$ with the $2 b_{1}$ $\mathrm{MO}$ with a positive sign and with the $4 \mathrm{~b}_{1}-\mathrm{MO}$ with a negative sign. The mixing of the $3 \mathrm{~b}_{1}$ with the $2 b_{1}$ and with the $1 b_{1} M O$ with a negative sign decreases the coefficient at the exocyclic carbon in the latter two MOs, and hence decreases the ipso-exo $\pi$-bond order. Similar effects can be seen at the CASSCF level, although the rotations among the occupied MOs distort the picture. In the UHF orbitals the $3 b_{1}$ MO seems to indicate transfer of spin from the exocyclic MOP to the ring, but the very pronounced spin polarization, which is very clearly visible (also in the $a_{2}-M O s$ which are not shown), over emphasizes this effect. 


\section{Results of Pariser-Parr-Pople (PPP) Calculations on the Rotational Barriers in the Allyl Cation, Radical, and Anion}

Due to the zero-differential overlap (ZDO) approximation in PPP theory, the $J$ and $K$ integrals in equations $1-3$ of the manuscript can be broken down into contributions from $\gamma_{11}$ (repulsion energy between two electrons in the same $2 \mathrm{p}-\mathrm{AO}$ ), $\gamma_{12}$ (repulsion energy between two electrons in neighboring $2 \mathrm{p}$-AOs), and $\gamma_{13}$ (repulsion energy between two electrons in 2p-AOs that are separated by two bonds). We assume that $\gamma_{11}$ is the same at planar ( $\mathrm{pl}$ ) and in the twisted (tw) geometries, but the two-center integrals can be different at these two geometries.. At a twisted geometry, one has to distinguish the situation where two p-AOs are parallel (i.e. in the $\pi$-bond) from that where the two p-AOs are perpendicular. We denote the latter type of Coulomb repulsion energies by the superscript $t w\left(\gamma_{12}^{t w}, \gamma_{13}^{t w}\right)$.

Using the Hückel MOs for planar and twisted allyl and the ZDO approximation, the $J$ and $K$-integrals over MOs in eqns. $1-3$ can be broken down into electron repulsion integrals between AOs in the planar and twisted geometries as follows:

$$
\begin{array}{ll}
J_{11}^{p l}=\left(3 \cdot \gamma_{11}+4 \cdot \gamma_{12}^{p l}+\gamma_{13}^{p l}\right) / 8 ; & J_{11}^{t w}=\left(4 \cdot \gamma_{11}+4 \cdot \gamma_{12}^{t w}\right) / 8 \\
J_{12}^{p l}=\left(2 \cdot \gamma_{11}+4 \cdot \gamma_{12}^{p l}+2 \cdot \gamma_{13}^{p l}\right) / 8 & J_{12}^{t w}=\left(4 \cdot \gamma_{12}^{t w}+4 \cdot \gamma_{13}^{t w}\right) / 8 \\
K_{12}^{p l}=\left(2 \cdot \gamma_{11}-2 \cdot \gamma_{13}^{p l}\right) / 8 ; & K_{12}^{t w}=0 \\
J_{22}^{p l}=\left(4 \cdot \gamma_{11}+4 \cdot \gamma_{13}^{p l}\right) / 8 ; & J_{22}^{t w}=\gamma_{11}
\end{array}
$$

In order to keep the evaluations of the $J$ and $K$ integrals in eqns. $4-7$ as simple as possible, we calculated all of these $\gamma_{s}$ at the geometries of the planar and twisted allyl cation (where the twisted $\mathrm{CH}_{2}$ group is planar). We used the minimal STO-3G basis set, which assures, for example, that $\gamma_{11}$ is the same in the anion, cation and radical. Perhaps surprisingly, but happily, the Hartree-Fock (HF)/STO-3G rotational barriers (calculated at the structures of planar $\mathbf{A}^{+}$and $\mathbf{A}^{-}$and at the HF/STO-3G transition states for rotation, are in reasonable agreement with those from the above W1BD calculations (34.4 and 25.3 for $\mathbf{A}^{+}$ and $\mathbf{A}^{-}$from HF/STO-3G vs. 32.8 and $20.8 \mathrm{kcal} / \mathrm{mol}$ from W1BD).

For $\mathbf{A}^{\bullet}$ things are a bit more complicated. The unrestricted (U)HF procedure overestimates the stabilizing effect of spin polarization in the planar radical and thus overestimates its barrier to rotation; whereas, the restricted open-shell (RO)HF method underestimates the barrier, largely due to the penchant of this method to localize the unpaired electron on one of the two $\mathrm{CH}_{2}$ groups. ${ }^{1}$ In fact, ROHF calculations with small basis sets find that the optimized geometry of even the planar allyl radical to consist of a ${ }^{\circ} \mathrm{CH}_{2}$ group attached to a localized ethene moiety by a C-C single bond. ${ }^{2}$

Fortunately, it is possible to sail safely between the Scylla of UHF and the Charybdis of ROHF $^{1}$ by performing a small CASSCF calculation, for which configurations are generated by distributing 3 electrons among the $3 \pi$ MOs. A (3/3)CASSCF/STO-3G calculation gives a 
rotational barrier for $\mathbf{A}^{\bullet}$ of $13.7 \mathrm{kcal} / \mathrm{mol}$, which is very close to the value of $14.7 \mathrm{kcal} / \mathrm{mol}$ computed at the W1BD level.

It is certainly worth noting that an analogous (2/3)CASSCF calculation for $\mathbf{A}^{+}$and (4/3)CASSCF for $\mathbf{A}^{-}$with the STO-3G basis set gives barriers $(31.3$ and $21.7 \mathrm{kcal} / \mathrm{mol}$, respectively) that are even closer to the W1BD barriers $(32.8$ and $20.8 \mathrm{kcal} / \mathrm{mol}$, respectively). This agreement shows that these calculated barriers depend very little on the level of sophistication of the methods that are used to compute them and thus instills a degree of confidence in our simple model calculations with the STO-3G basis set.

The STO-3G values for the $\gamma \mathrm{s}$ are listed in the top row of Table S-1. These values allow us to compute the $J_{\mathrm{s}}$ and $K \mathrm{~s}$ in equations 4-7 above. These Coulomb and exchange integrals are given in the second row of Table S-1. The differences between the $J_{\mathrm{s}}$ and $K \mathrm{~s}$ that are needed to compute the changes in the electron repulsion terms on twisting, using eqns. $1-3$, are provided in the third row, while the differences in electron repulsion between the planar and the twisted forms are listed in the bottom row of Table S-1.

Table S-1: STO-3G values of the electron repulsion integrals (in $\mathrm{kcal} / \mathrm{mol}$ ) between AOs (first row) and between MOs (second row), in the planar (pl) and twisted (tw) allyl systems, calculated at the CAS(2,3)/3-21G geometry of the allyl cation. The third row gives the differences between the $J_{\mathrm{s}}$ and $K s$ in the planar and twisted geometries.

\begin{tabular}{|c|c|c|c|c|c|c|c|c|}
\hline integral & $\gamma_{11}$ & $\gamma_{12}^{p l}$ & $\gamma_{13}^{p l}$ & $\gamma_{12}^{t w}$ & $\gamma_{12}^{t w}$ & $\gamma_{13}^{t w}$ & & \\
\hline value & 422.2 & 212.9 & 133.1 & 221.8 & 196.9 & 128.3 & & \\
\hline integral & $J_{11}^{p l}$ & $J_{11}^{t w}$ & $J_{12}^{p l}$ & $J_{12}^{t w}$ & $K_{12}^{p l}$ & $K_{12}^{t w}$ & $J_{22}^{p l}$ & $J_{22}^{t w}$ \\
\hline value & 281.4 & 317.5 & 245.3 & 162.6 & 72.3 & 0 & 277.6 & 422.2 \\
\hline difference & \multicolumn{2}{|c|}{$J_{11}^{t w}-J_{11}^{p l}$} & \multicolumn{2}{|c|}{$J_{12}^{t w}-J_{12}^{p l}$} & \multicolumn{2}{|c|}{$K_{12}^{t w}-K_{12}^{p l}$} & \multicolumn{2}{|c|}{$J_{22}^{t w}-J_{22}^{p l}$} \\
\hline value & \multicolumn{2}{|c|}{36.1} & \multicolumn{2}{|c|}{-82.6} & \multicolumn{2}{|c|}{-72.3} & \multicolumn{2}{|c|}{144.6} \\
\hline$\Delta_{\text {elrep }^{a}}$ & \multicolumn{2}{|c|}{ cation } & \multicolumn{2}{|c|}{ radical } & \multicolumn{2}{|c|}{ anion } & & \\
\hline value & \multicolumn{2}{|c|}{36.1} & \multicolumn{2}{|c|}{-56.8} & \multicolumn{2}{|c|}{-5.2} & & \\
\hline
\end{tabular}

$a$ difference in electron repulsion between the planar and the twisted form, see eq. 1-3

Due to the approximations that we made, the values of the numbers in the last row of Table S-1 are not even close to being quantitatively correct; but this simple model has the virtue of revealing why the allyl cation is calculated to have a higher barrier to rotation than the anion and why the anion has a higher barrier than the radical. The Coulombic repulsion $\left(J_{11}\right)$ between the pair of electrons in the bonding MO is higher in the more localized ethylenic bonding $\pi \mathrm{MO}$ in the twisted geometry; and this tends to favor the planar geometry for $\mathbf{A}^{+}$, $\mathbf{A}^{*}$, and $\mathbf{A}^{-}$. In $\mathbf{A}^{\cdot}$ the Coulombic repulsion between this pair of electrons and the electron in 
the NBMO $\left(2 J_{12}-K_{12}\right)$ is far smaller in the twisted geometry, where the unpaired electron is localized on the twisted $\mathrm{CH}_{2}$ group. This localization favors the twisted geometry not only in $\mathbf{A}^{\bullet}$ but also in $\mathbf{A}^{-}$. However, in $\mathbf{A}^{-}$both nonbonding electrons become localized in the $\mathrm{AO}$ on the twisted carbon; and their mutual Coulombic repulsion destabilizes the twisted geometry.

In order to examine the consequences of the approximations we made in our PPP-type calculations, there are mainly two types of possible errors to assess critically: (a) the differences in geometries between the cation, the radical, and the anion, and (b) the fact that the bonding and nonbonding MOs for the three species are not the same.

Concerning the geometries, the effect of the relaxation from the cation geometry on the rotational barrier is relatively small in the radical $(2.2 \mathrm{kcal} / \mathrm{mol}$ at the planar, $4.8 \mathrm{kcal} / \mathrm{mol}$ at the twisted geometry), but much more pronounced in the anion $(7.8 \mathrm{kcal} / \mathrm{mol}$ at the planar, 31.7 $\mathrm{kcal} / \mathrm{mol}$ at the twisted geometry). At the planar geometry the stabilization is mainly due to the opening of the angle which decreases the 1,3-antibonding interaction in the NBMO; whereas, at the twisted geometry, the strong pyramidalization of the rotated $\mathrm{CH}_{2}{ }^{-}$group is primarily responsible for the even stronger stabilization.

While the effect of the changing angle could easily be accommodated in our above simple model (but would not change much), the consequences of pyramidalization on the PPP-type electron repulsion integrals is more difficult to model, because, instead of simple p-AOs, sp hybrids are involved, which cannot readily be dealt with in the PPP-approximation. However, we can assume that this pyramidalization decreases the repulsion between the pair of nonbonding and the pair of $\pi$-bonding electrons at the twisted geometry, because the main lobe points away from the $\pi$-bond, i.e. $\Delta_{\text {elrep }}\left(\mathbf{A}^{-}\right)$should be more negative than indicated in Table 2.

Obviously, allowing the AOs to shrink or expand as a function of the charge of the system will affect the electron repulsion integrals quite profoundly. However, the fact that the rotational barriers are quite insensitive to the basis set $^{16}$ indicates that the planar and the twisted structure are similarly affected by this orbital relaxation, so the $\Delta_{\text {elrep }}$ should not change greatly as a consequence of it.

To account properly for all the above factors, we have computed the $J \mathrm{~s}$ and $K \mathrm{~s}$ directly in the basis of the SCF MOs, and we augmented the basis set to $6-311++\mathrm{G}^{* *}$, to allow for optimal orbital relaxation as the charge of the allyl system changes. By this approach the hyperconjugation in the twisted structures, which is quite pronounced (as can be seen from the SCF MOs shown in Figure S-1 of the Supporting Information), is also taken into account. Table S-2 shows the values we obtained for these integrals calculated at the SCF level.

Of course the $\Delta_{\text {elrep }}$ values change from those computed at the frozen geometries of the planar and twisted allyl cation with the STO-3G basis set. However, the order of these $\Delta_{\text {elrep }}$ values is still the same. Electron repulsion increases the barrier to $\mathrm{CH}_{2}$ group rotation in the 
cation, increases the barrier in the anion, but by less than in the cation, and decreases the barrier for the radical.

Table S-2: Values of the $J$ - and $K$-integrals for $\mathbf{A}^{+}, \mathbf{A}^{\bullet}$, and $\mathbf{A}^{-}$, calculated in the basis of the $\mathrm{SCF}$ MOs with the 6-311++ $\mathrm{G}^{* *}$ basis set at the CAS(x,3)/3-21G-optimized geometries $(\mathrm{x}=2,3$, or 4 electrons, respectively).

\begin{tabular}{cccccccccc}
\hline & $J_{11}^{p l}$ & $J_{11}^{t w}$ & $J_{12}^{p l}$ & $J_{12}^{t w}$ & $K_{12}^{p l}$ & $K_{12}^{t w}$ & $J_{22}^{p l}$ & $J_{22}^{t w}$ & $\Delta_{\text {elrep }}$ \\
\hline $\mathbf{A}^{+}$ & 259.7 & 272.3 & - & - & - & - & - & - & 12.6 \\
$\mathbf{A}^{\bullet}$ & 246.3 & 260.9 & 212.0 & 159.2 & 58.7 & 1.1 & - & - & -33.5 \\
$\mathbf{A}^{-}$ & 234.5 & 236.9 & 198.0 & 151.5 & 56.8 & 2.1 & 198.4 & 277.4 & 4.8 \\
\hline \hline
\end{tabular}

Even with the correct geometries and a big basis set, the values of $\Delta_{\text {elrep }}$ in Table S-2 focus on a small fraction of the total electron repulsion energies. In addition, there are other terms that contribute to the total energies (expectation values from the core hamiltonian, nuclear repulsion), which also change on twisting. Therefore, it is not to be expected that the $\Delta_{\text {elrep }}$ values in Table S-2 should reproduce exactly the differences in rotational barriers for the allyl cation, radical, and anion in Table 1 of the text. Nevertheless, the energies in Table S-2 allow us to explain qualitatively the origin of the differences between the rotational barriers in the allyl cation, radical, and anion in Table 1.

\section{References}

1. Bally, T.; Borden, W. T., Rev. Comput. Chem. 2007, 13, 1.

2. For a discussion, see: Davidson, E. R.; Borden, W. T., J. Phys. Chem. 1983, 87, 4783. 
Total energies, thermal corrections, and cartesian coordinates of all stationary points located in this study.

\begin{tabular}{|c|c|c|c|}
\hline \multicolumn{4}{|c|}{$\begin{array}{l}\text { 1. planar allyl cation } \\
* * * * * * * * * * * * * * * * * * * * *\end{array}$} \\
\hline \multicolumn{4}{|c|}{$\begin{array}{l}============================================ \\
\mathrm{HF} / \mathrm{STO}-3 \mathrm{G}(\mathrm{C} 2 \mathrm{~V})\end{array}$} \\
\hline \multicolumn{4}{|c|}{$\begin{array}{l}\text { State }=1-\mathrm{A} 1 \backslash \mathrm{HF}=-114.8095268 \\
\text { Potential energy minimum }\end{array}$} \\
\hline & $\mathrm{X}$ & $\mathrm{Y}$ & Z \\
\hline 6 & 0.000000 & 1.191981 & -0.210808 \\
\hline 6 & 0.000000 & 0.000000 & 0.493192 \\
\hline 6 & 0.000000 & -1.191981 & -0.210808 \\
\hline 1 & 0.000000 & 2.164291 & 0.305367 \\
\hline 1 & 0.000000 & 1.218067 & -1.310583 \\
\hline 1 & 0.000000 & 0.000000 & 1.577570 \\
\hline 1 & 0.000000 & -2.164291 & 0.305367 \\
\hline 1 & 0.000000 & -1.218067 & -1.310583 \\
\hline
\end{tabular}

State $=1-\mathrm{A} 1 \backslash \mathrm{HF}=-114.8447245$

Potential energy minimum

\begin{tabular}{rrrr} 
& $\mathrm{X}$ & \multicolumn{1}{c}{$\mathrm{Y}$} & \multicolumn{1}{c}{$\mathrm{Z}$} \\
6 & 0.000000 & 1.203689 & -0.212722 \\
6 & 0.000000 & 0.000000 & 0.495972 \\
6 & 0.000000 & -1.203689 & -0.212722 \\
1 & 0.000000 & 2.171697 & 0.305916 \\
1 & 0.000000 & 1.222661 & -1.310165 \\
1 & 0.000000 & 0.000000 & 1.585328 \\
1 & 0.000000 & -2.171697 & 0.305916 \\
1 & 0.000000 & -1.222661 & -1.310165
\end{tabular}

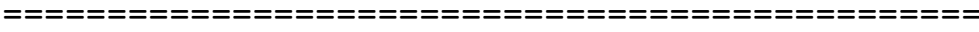
$\mathrm{HF} / 3-21 \mathrm{G}(\mathrm{C} 2 \mathrm{v})$

State $=1-\mathrm{A} 1 \backslash \mathrm{HF}=-115.542137$

Potential energy minimum

\begin{tabular}{rrrr} 
& $\mathrm{X}$ & \multicolumn{1}{c}{$\mathrm{Y}$} & \multicolumn{1}{c}{$\mathrm{Z}$} \\
6 & 0.000000 & 1.182332 & -0.208313 \\
6 & 0.000000 & 0.000000 & 0.484380 \\
6 & 0.000000 & -1.182332 & -0.208313 \\
1 & 0.000000 & 2.126975 & 0.302405 \\
1 & 0.000000 & 1.209567 & -1.283153 \\
1 & 0.000000 & 0.000000 & 1.554969 \\
1 & 0.000000 & -2.126975 & 0.302405 \\
1 & 0.000000 & -1.209567 & -1.283153
\end{tabular}

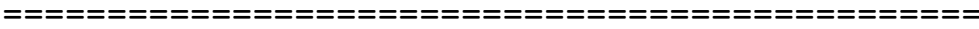
$\operatorname{CAS}(2,3) / 3-21 \mathrm{G}(\mathrm{C} 2 \mathrm{v})$

State=1-A $1 \backslash \mathrm{HF}=-115.5693337$

Potential energy minimum $\mathrm{X}$

Y Z

$\begin{array}{rrrr}6 & 0.000000 & 1.188906 & -0.208825 \\ 6 & 0.000000 & 0.000000 & 0.485107 \\ 6 & 0.000000 & -1.188906 & -0.208825 \\ 1 & 0.000000 & 2.132593 & 0.302355 \\ 1 & 0.000000 & 1.213408 & -1.283076\end{array}$




$\begin{array}{rrrr}1 & 0.000000 & 0.000000 & 1.556696 \\ 1 & 0.000000 & -2.132593 & 0.302355 \\ 1 & 0.000000 & -1.213408 & -1.283076\end{array}$

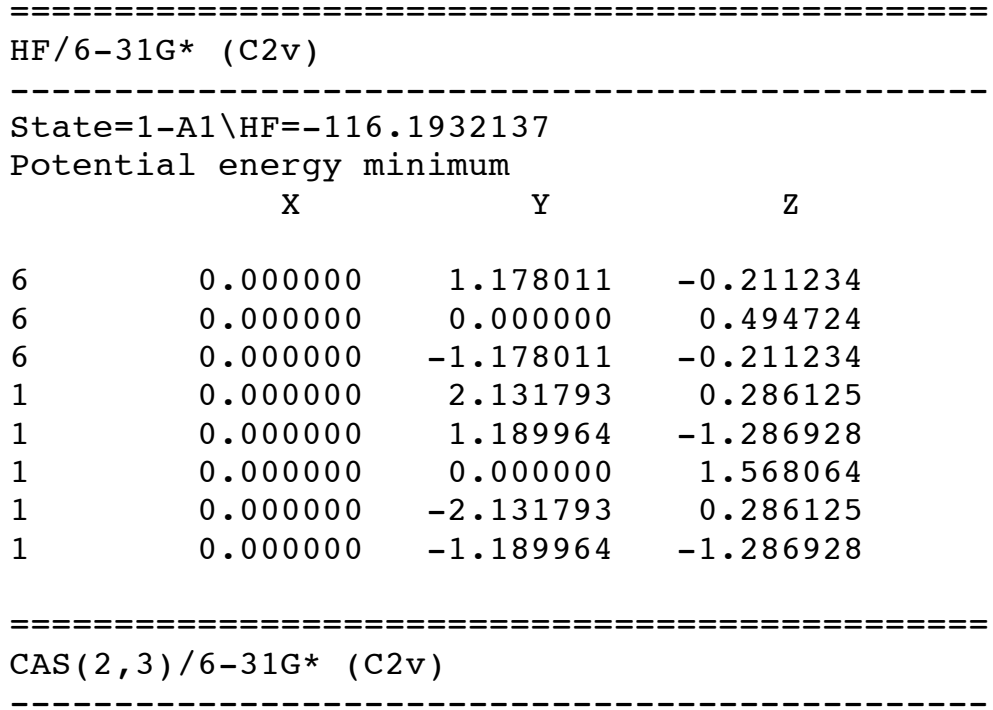

State $=1-\mathrm{A} 1 \backslash \mathrm{HF}=-116.2227009$

Potential energy minimum

single-point energy with the 6-311++G** basis: $\mathrm{HF}=-116.2511931$

$\mathrm{X}$

$\mathrm{Y}$

Z

$\begin{array}{rrrr}6 & 0.000000 & 1.186608 & -0.210450 \\ 6 & 0.000000 & 0.000000 & 0.490818 \\ 6 & 0.000000 & -1.186608 & -0.210450 \\ 1 & 0.000000 & 2.136372 & 0.293542 \\ 1 & 0.000000 & 1.201496 & -1.285892 \\ 1 & 0.000000 & 0.000000 & 1.565192 \\ 1 & 0.000000 & -2.136372 & 0.293542 \\ 1 & 0.000000 & -1.201496 & -1.285892\end{array}$

\begin{tabular}{|c|c|c|c|}
\hline \multicolumn{4}{|c|}{ B3LYP/6-31G(2df,p) (C1) (from G4 calculation) } \\
\hline \multicolumn{4}{|c|}{$\begin{array}{l}\text { State }=1-A \backslash E(R B 3 L Y P)=-116.982152165 \\
\text { potential energy minimum } \\
\text { G4-energy: }-116.861132 \text { (enthaply:-116.860188) }\end{array}$} \\
\hline & $\mathrm{X}$ & $\mathrm{Y}$ & $\mathrm{z}$ \\
\hline & 1.187458 & -0.211150 & -0.000008 \\
\hline & 0.000000 & 0.494846 & -0.000006 \\
\hline & 2.152403 & 0.291880 & -0.000020 \\
\hline & 1.196126 & -1.299589 & 0.000009 \\
\hline & 0.000001 & 1.580142 & -0.000020 \\
\hline & -1.187459 & -0.211150 & 0.000014 \\
\hline & -1.196123 & -1.299589 & 0.000023 \\
\hline & -2.152403 & 0.291880 & 0.000008 \\
\hline \multirow{2}{*}{\multicolumn{4}{|c|}{$\begin{array}{l}==================================== \\
3 \mathrm{LYP} / \mathrm{cC}-\mathrm{pVTZ}+\mathrm{d} \text { (C1) (from W1BD calculation) }\end{array}$}} \\
\hline & & & \\
\hline \multicolumn{4}{|c|}{ 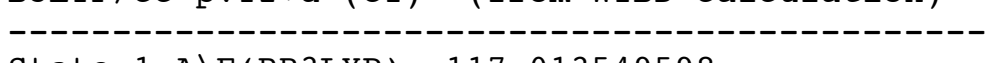 } \\
\hline \multirow{2}{*}{\multicolumn{4}{|c|}{$\begin{array}{l}\text { State }=1-A \backslash E(R B 3 L Y P)=-117.013549598 \\
\text { potential energy minimum } \\
\text { W1BD-energy: }-116.912513 \text { (enthaply:-116.911569) }\end{array}$}} \\
\hline & & & \\
\hline & $\mathrm{X}$ & $\mathrm{Y}$ & $\mathrm{Z}$ \\
\hline & 1.184679 & -0.210400 & -0.000007 \\
\hline & -0.000001 & 0.492169 & -0.000006 \\
\hline & 2.144835 & 0.293886 & -0.000020 \\
\hline
\end{tabular}




$\begin{array}{lrrr}1 & 1.193474 & -1.295164 & 0.000009 \\ 1 & 0.000002 & 1.574353 & -0.000020 \\ 6 & -1.184679 & -0.210401 & 0.000013 \\ 1 & -1.193468 & -1.295164 & 0.000023 \\ 1 & -2.144835 & 0.293886 & 0.000009\end{array}$

$=====================================$ B3LYP / 6-311++G** (C1)

State $=1-A \backslash E($ RB3LYP $)=-117.001552484$

Potential energy minimum

\begin{tabular}{lrrr} 
& $X$ & \multicolumn{1}{c}{$Y$} & $Z$ \\
6 & 1.188756 & -0.210721 & -0.000007 \\
6 & -0.000011 & 0.492247 & -0.000006 \\
1 & 2.149737 & 0.297291 & -0.000019 \\
1 & 1.201926 & -1.298085 & 0.000005 \\
1 & 0.000022 & 1.576804 & -0.000018 \\
6 & -1.188758 & -0.210731 & 0.000012 \\
1 & -1.201888 & -1.298091 & 0.000029 \\
1 & -2.149722 & 0.297310 & 0.000005
\end{tabular}

2. twisted allyl cation

$* * * * * * * * * * * * * * * * * * * * * * *$

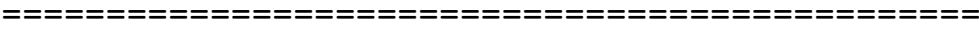
$\mathrm{HF} / \mathrm{STO}-3 \mathrm{G}$ (Cs)

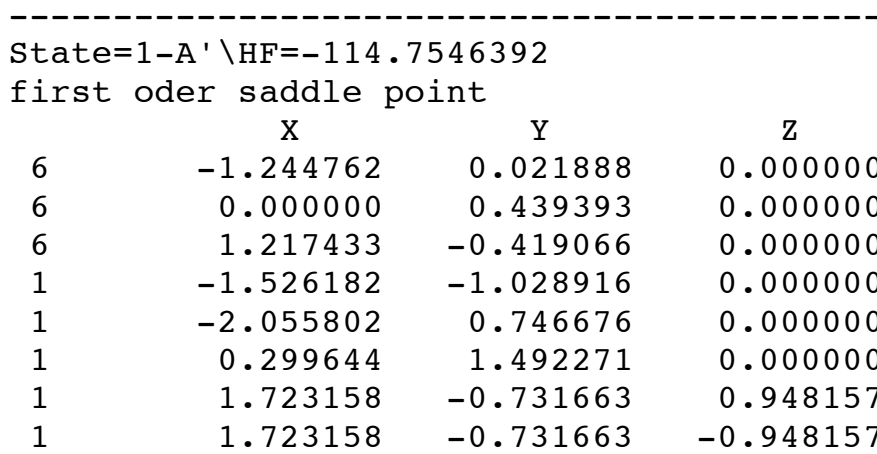

\begin{tabular}{|c|c|c|c|}
\hline \multicolumn{4}{|c|}{$\operatorname{CAS}(2,3) / \mathrm{STO}-3 \mathrm{G}$ (Cs) } \\
\hline \multicolumn{4}{|c|}{ State $=1-\mathrm{A} 1 \backslash \mathrm{HF}=-114.8447245$} \\
\hline & $\mathrm{X}$ & $\mathrm{Y}$ & $\mathrm{Z}$ \\
\hline 6 & -1.268517 & -0.012711 & 0.000000 \\
\hline 6 & 0.000000 & 0.437215 & 0.000000 \\
\hline 6 & 1.237073 & -0.391749 & 0.000000 \\
\hline 1 & -1.521806 & -1.068167 & 0.000000 \\
\hline 1 & -2.084174 & 0.702859 & 0.000000 \\
\hline 1 & 0.271086 & 1.499888 & 0.000000 \\
\hline 1 & 1.761777 & -0.665554 & 0.950248 \\
\hline 1 & 1.761777 & -0.665554 & -0.950248 \\
\hline
\end{tabular}

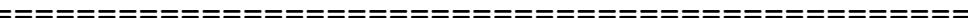

$\mathrm{HF} / 3-21 \mathrm{G}$ (Cs)

State $=1-A^{\prime} \backslash \mathrm{HF}=-115.4878209$

first oder saddle point

$$
\mathrm{X}
$$

$\begin{array}{rrrr}6 & -1.241287 & -0.030039 & 0.000000 \\ 6 & 0.000000 & 0.407761 & 0.000000 \\ 6 & 1.220514 & -0.351885 & 0.000000 \\ 1 & -1.496335 & -1.071677 & 0.000000\end{array}$




$\begin{array}{rrrr}1 & -2.052811 & 0.668642 & 0.000000 \\ 1 & 0.236366 & 1.473588 & 0.000000 \\ 1 & 1.718709 & -0.612788 & 0.922519 \\ 1 & 1.718709 & -0.612788 & -0.922519\end{array}$

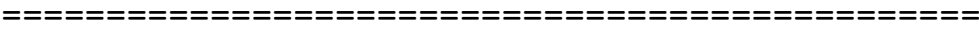
$\operatorname{CAS}(2,3) / 3-21 \mathrm{G}$ (Cs)

State $=1-\mathrm{A}^{\prime} \backslash \mathrm{HF}=-115.5170873$ lout

first oder saddle point

single-point energy with the 6-311++G** basis: $\mathrm{HF}=-116.2511931$

$\mathrm{X}$

$\mathrm{Y}$

$\mathrm{Z}$

$\begin{array}{rrrr}6 & -1.246060 & -0.063509 & 0.000000 \\ 6 & 0.000000 & 0.423265 & 0.000000 \\ 6 & 1.228141 & -0.328159 & 0.000000 \\ 1 & -1.463372 & -1.113119 & 0.000000 \\ 1 & -2.076672 & 0.611615 & 0.000000 \\ 1 & 0.202809 & 1.494214 & 0.000000 \\ 1 & 1.722375 & -0.591147 & 0.923291 \\ 1 & 1.722375 & -0.591147 & -0.923291\end{array}$

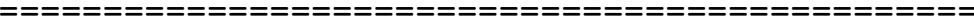
$\mathrm{HF} / 6-31 \mathrm{G} *$ (CS)

State=1-A $\backslash \mathrm{HF}=-116.1389889$

first oder saddle point $\mathrm{X}$ $\mathrm{Y}$ $\mathrm{Z}$

$\begin{array}{rrrr}6 & -1.241877 & -0.025842 & 0.000000 \\ 6 & 0.000000 & 0.416074 & 0.000000 \\ 6 & 1.218755 & -0.360178 & 0.000000 \\ 1 & -1.493662 & -1.070332 & 0.000000 \\ 1 & -2.053930 & 0.675106 & 0.000000 \\ 1 & 0.248985 & 1.477562 & 0.000000 \\ 1 & 1.718670 & -0.631331 & 0.919900 \\ 1 & 1.718670 & -0.631331 & -0.919900\end{array}$

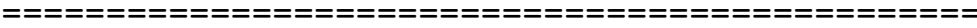
$\operatorname{CAS}(2,3) / 6-31 \mathrm{G} *$ (Cs)

State $=1-A^{\prime} \backslash \mathrm{HF}=-116.1673194$

first oder saddle point

single-point energy with the 6-311++G** basis: $\mathrm{HF}=-116.1967493: \mathrm{q}$ !

$\mathrm{X}$

-1.248603
0.000000
1.227306
-1.467309
-2.076956
0.220841
1.725602
1.725602

$-1.248603$

0.427501

$-0.336313$

$-1.108296$

0.623617

1.493768

$-0.606876$

$-0.606876$
Z

0.000000

0.000000

0.000000

0.000000

0.000000

0.000000

0.920367

$-0.920367$

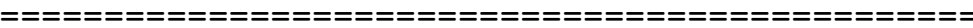
B3LYP/6-31G(2df, P) (C1) (from G4 calculation)

State $=1-A \backslash E($ RB3LYP $)=-116.923658577$

second oder saddle point (a minor negative 
frequency comes from the fixed C-C-C angle) G4-energy:-116.809958 (enthaply:-116.809013)

\begin{tabular}{rrrr} 
& \multicolumn{1}{c}{$\mathrm{X}$} & \multicolumn{1}{c}{$\mathrm{Y}$} \\
6 & -1.207292 & -0.161242 & -0.000002 \\
6 & 0.058472 & 0.490119 & 0.000019 \\
1 & -1.726025 & -0.429463 & -0.930278 \\
1 & -1.726141 & -0.429490 & 0.930198 \\
1 & 0.059187 & 1.588928 & -0.000009 \\
6 & 1.166189 & -0.263714 & 0.000000 \\
1 & 1.153927 & -1.347404 & 0.000032 \\
1 & 2.134843 & 0.226449 & -0.000043
\end{tabular}

$=================================$ B3LYP/cC-pVTZ+d (C1) (from W1BD calculation)

State $=1-A \backslash E(R B 3 L Y P)=-116.955847422$

second oder saddle point (a minor negative frequency comes from the fixed C-C-C angle) W1BD-energy:-116.861049 (enthaply:-116.860105) $\mathrm{X}$ $\mathrm{Y}$ $\mathrm{Z}$

$\begin{array}{rrrr}6 & -1.204707 & -0.160919 & -0.000002 \\ 6 & 0.057804 & 0.487461 & 0.000017 \\ 1 & -1.719741 & -0.425788 & -0.928587 \\ 1 & -1.719844 & -0.425813 & 0.928515 \\ 1 & 0.061974 & 1.584394 & -0.000008 \\ 6 & 1.163390 & -0.262666 & 0.000000 \\ 1 & 1.150315 & -1.343339 & 0.000029 \\ 1 & 2.128377 & 0.227291 & -0.000038\end{array}$

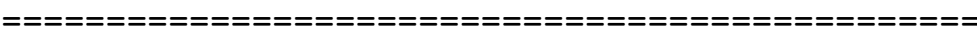
B3LYP / 6-311++G** (C1)

State $=1-\mathrm{A} \backslash \mathrm{E}(\mathrm{RB} 3 \mathrm{LYP})=-116.943563554$

First order saddle point

$\mathrm{x}$

$-1.207766$

0.058106

$-1.723569$

$-1.723685$

0.060824

1.166053

1.154946

2.133120
$\mathrm{Y}$

$-0.161942$

0.489889

$-0.427581$

$-0.427611$

1.588891

$-0.263680$

$-1.346788$

0.227487
$-0.000003$

0.000019

$-0.931018$

0.930937

$-0.000008$

0.000000

0.000032

$-0.000044$

3. planar allyl radical

$* * * * * * * * * * * * * * * * * * * * * * *$

\begin{tabular}{|c|c|c|c|}
\hline \multicolumn{4}{|c|}{$\mathrm{UHF} / \mathrm{STO}-3 \mathrm{G}$ (C2v) } \\
\hline \multicolumn{4}{|c|}{$\begin{array}{l}\text { State }=2-\mathrm{A} 2 \backslash \mathrm{HF}=-115.0542911 \backslash \mathrm{S} 2=1.101186 ? \\
\text { Potential energy minimum }\end{array}$} \\
\hline & $\mathrm{x}$ & $\mathrm{Y}$ & $\mathrm{Z}$ \\
\hline 6 & 0.000000 & 1.240947 & -0.201886 \\
\hline 6 & 0.000000 & 0.000000 & 0.457930 \\
\hline 6 & 0.000000 & -1.240947 & -0.201886 \\
\hline 1 & 0.000000 & 2.171378 & 0.348054 \\
\hline 1 & 0.000000 & 1.301571 & -1.281704 \\
\hline 1 & 0.000000 & 0.000000 & 1.542344 \\
\hline 1 & 0.000000 & -2.171378 & 0.348054 \\
\hline 1 & 0.000000 & -1.301571 & -1.281704 \\
\hline
\end{tabular}


ROHF/STO-3G (C2V symmetry enforced)

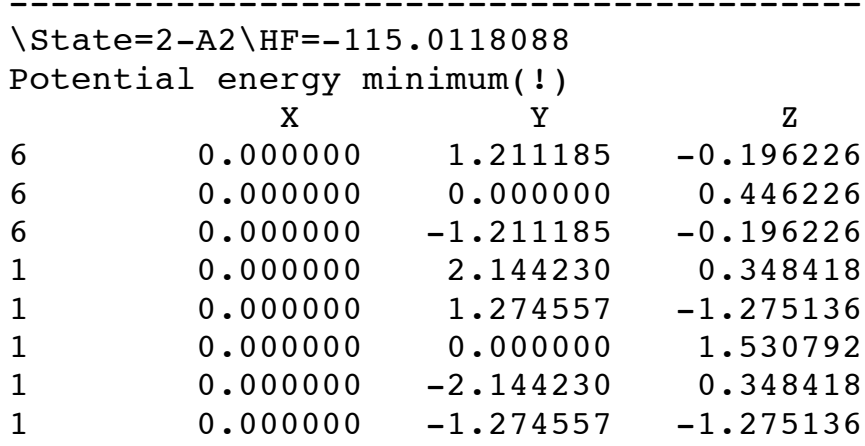

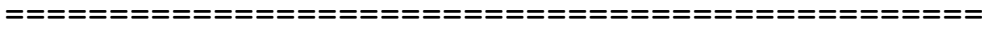
ROHF/STO-3G (Cs symmetry enforced)

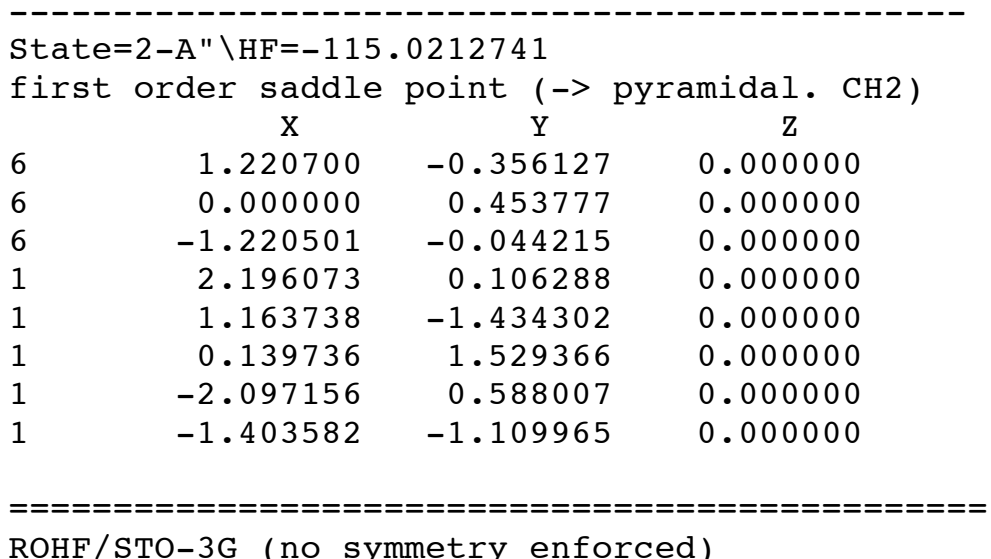

ROHF/STO-3G (no symmetry enforced)

State $=2-\mathrm{A} \backslash \mathrm{HF}=-115.0212743$

potential energy minimum

$$
\mathrm{X}
$$

$\mathrm{Y}$

$\begin{array}{rrr}1.258313 & 0.184681 & -0.008545 \\ -0.062551 & -0.449456 & -0.000431 \\ -1.202792 & 0.211864 & 0.001095 \\ 2.159955 & -0.407704 & 0.027683 \\ 1.350219 & 1.260281 & 0.015408 \\ -0.072283 & -1.534085 & 0.000249 \\ -2.158115 & -0.293720 & 0.003742 \\ -1.237599 & 1.292694 & 0.000204\end{array}$

作 $\operatorname{CAS}(2,3) / \mathrm{STO}-3 \mathrm{G}(\mathrm{C} 2 \mathrm{~V})$

State $=2-\mathrm{A} 2 \backslash \mathrm{HF}=-115.0748693$

Potential energy minimum

\begin{tabular}{lcrr} 
& $\mathrm{X}$ & \multicolumn{1}{c}{$\mathrm{Y}$} & \multicolumn{1}{c}{$\mathrm{Z}$} \\
6 & 0.000000 & 1.237225 & -0.200605 \\
6 & 0.000000 & 0.000000 & 0.454362 \\
6 & 0.000000 & -1.237225 & -0.200605 \\
1 & 0.000000 & 2.165958 & 0.350546 \\
1 & 0.000000 & 1.300247 & -1.279153 \\
1 & 0.000000 & 0.000000 & 1.538296 \\
1 & 0.000000 & -2.165958 & 0.350546 \\
1 & 0.000000 & -1.300247 & -1.279153
\end{tabular}




\begin{tabular}{|c|c|c|c|}
\hline \multicolumn{4}{|c|}{$\begin{array}{l}============= \\
\mathrm{UHF} / 3-21 \mathrm{G} \quad(\mathrm{C} 2 \mathrm{v})\end{array}$} \\
\hline \multicolumn{4}{|c|}{ 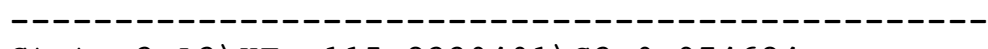 } \\
\hline \multicolumn{4}{|c|}{ State $=2-\mathrm{A} 2 \backslash \mathrm{HF}=-115.8230401 \backslash \mathrm{S} 2=0.974684$} \\
\hline \multicolumn{4}{|c|}{ Potential energy minimum } \\
\hline & $\mathrm{X}$ & $\mathrm{Y}$ & $\mathrm{Z}$ \\
\hline 6 & 0.000000 & 1.227626 & -0.198661 \\
\hline 6 & 0.000000 & 0.000000 & 0.450220 \\
\hline 6 & 0.000000 & -1.227626 & -0.198661 \\
\hline 1 & 0.000000 & 2.149339 & 0.349250 \\
\hline 1 & 0.000000 & 1.289221 & -1.270853 \\
\hline 1 & 0.000000 & 0.000000 & 1.525816 \\
\hline 1 & 0.000000 & -2.149339 & 0.349250 \\
\hline 1 & 0.000000 & -1.289221 & -1.270853 \\
\hline \multicolumn{4}{|c|}{ 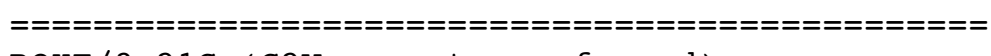 } \\
\hline \multicolumn{4}{|c|}{ ROHF/3-21G (C2V symmetry enforced) } \\
\hline \multicolumn{4}{|c|}{-ーーーーーーーーーーーーーーーーーーーーーーーーーーーーーーー } \\
\hline \multicolumn{4}{|c|}{ State $=2-\mathrm{A} 2 \backslash \mathrm{HF}=-115.7977668$} \\
\hline \multicolumn{4}{|c|}{ (no frequency calculation possible) } \\
\hline & $\mathrm{X}$ & $\mathrm{Y}$ & $\mathrm{Z}$ \\
\hline 6 & 0.000000 & 1.211195 & -0.195527 \\
\hline 6 & 0.000000 & 0.000000 & 0.443652 \\
\hline 6 & 0.000000 & -1.211195 & -0.195527 \\
\hline 1 & 0.000000 & 2.133673 & 0.349330 \\
\hline 1 & 0.000000 & 1.274154 & -1.266822 \\
\hline 1 & 0.000000 & 0.000000 & 1.519398 \\
\hline 1 & 0.000000 & -2.133673 & 0.349330 \\
\hline 1 & 0.000000 & -1.274154 & -1.266822 \\
\hline \multirow{2}{*}{\multicolumn{4}{|c|}{$\begin{array}{l}========================================== \\
\text { ROHF } / 3-21 \mathrm{G} \text { (Cs symmtery enforced) }\end{array}$}} \\
\hline & & & \\
\hline \multirow{2}{*}{\multicolumn{4}{|c|}{ 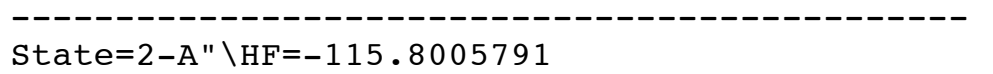 }} \\
\hline & & & \\
\hline \multicolumn{4}{|c|}{ Potential energy minimum } \\
\hline & $\mathrm{X}$ & $\mathrm{Y}$ & $\mathrm{Z}$ \\
\hline 6 & 1.217707 & -0.308615 & 0.000000 \\
\hline 6 & 0.000000 & 0.448746 & 0.000000 \\
\hline 6 & -1.217222 & -0.087474 & 0.000000 \\
\hline 1 & 2.172060 & 0.176327 & 0.000000 \\
\hline 1 & 1.193925 & -1.380410 & 0.000000 \\
\hline 1 & 0.096690 & 1.519793 & 0.000000 \\
\hline 1 & -2.100982 & 0.519831 & 0.000000 \\
\hline 1 & -1.364601 & -1.151484 & 0.000000 \\
\hline \multirow{2}{*}{\multicolumn{4}{|c|}{$\begin{array}{l}=========================================== \\
\operatorname{CAS}(2,3) / 3-21 \mathrm{G}(\mathrm{C} 2 \mathrm{v})\end{array}$}} \\
\hline & & & \\
\hline \multicolumn{4}{|c|}{ State $=2-\mathrm{A} 2 \backslash \mathrm{HF}=-115.8402426$} \\
\hline \multicolumn{4}{|c|}{ Potential energy minimum } \\
\hline & $\mathrm{X}$ & $\mathrm{Y}$ & $\mathrm{Z}$ \\
\hline 6 & 0.000000 & 1.228022 & -0.198329 \\
\hline 6 & 0.000000 & 0.000000 & 0.449128 \\
\hline 6 & 0.000000 & -1.228022 & -0.198329 \\
\hline 1 & 0.000000 & 2.148602 & 0.350020 \\
\hline 1 & 0.000000 & 1.290890 & -1.269710 \\
\hline 1 & 0.000000 & 0.000000 & 1.524561 \\
\hline 1 & 0.000000 & -2.148602 & 0.350020 \\
\hline 1 & 0.000000 & -1.290890 & -1.269710 \\
\hline
\end{tabular}




\begin{tabular}{|c|c|c|c|}
\hline \multicolumn{4}{|c|}{$\mathrm{UHF} / 6-31 \mathrm{G} * \quad(\mathrm{C} 2 \mathrm{v})$} \\
\hline \multicolumn{4}{|c|}{$\begin{array}{l}\text { State }=2-\mathrm{A} 2 \backslash \mathrm{HF}=-116.4680999 \backslash \mathrm{S} 2=0.972865 \\
\text { Potential energy minimum }\end{array}$} \\
\hline & $\mathrm{X}$ & $\mathrm{Y}$ & $\mathrm{Z}$ \\
\hline 6 & 0.000000 & 1.230851 & -0.198289 \\
\hline 6 & 0.000000 & 0.000000 & 0.448532 \\
\hline 6 & 0.000000 & -1.230851 & -0.198289 \\
\hline 1 & 0.000000 & 2.152790 & 0.352839 \\
\hline 1 & 0.000000 & 1.296108 & -1.271968 \\
\hline 1 & 0.000000 & 0.000000 & 1.526538 \\
\hline 1 & 0.000000 & -2.152790 & 0.352839 \\
\hline 1 & 0.000000 & -1.296108 & -1.271968 \\
\hline
\end{tabular}

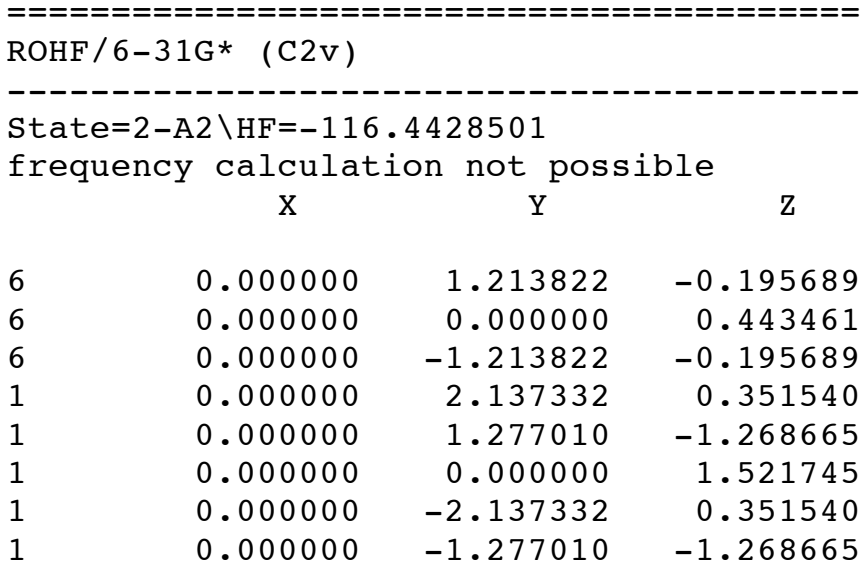

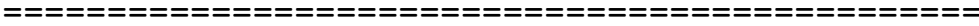
$\mathrm{ROHF} / 6-31 \mathrm{G} *(\mathrm{Cs})$

State $=2-\mathrm{A} " \backslash \mathrm{HF}=-116.4457928$

frequency calculation not possible $\mathrm{X}$

$$
\mathrm{Y}
$$

Z

$\begin{array}{rrrr}6 & 1.220634 & -0.306995 & 0.000000 \\ 6 & 0.000000 & 0.446577 & 0.000000 \\ 6 & -1.220367 & -0.088091 & 0.000000 \\ 1 & 2.175682 & 0.180320 & 0.000000 \\ 1 & 1.202434 & -1.380365 & 0.000000 \\ 1 & 0.093759 & 1.520589 & 0.000000 \\ 1 & -2.103216 & 0.524456 & 0.000000 \\ 1 & -1.370262 & -1.153947 & 0.000000\end{array}$

$==============================================$
$\operatorname{CAS}(2,3) / 6-31 \mathrm{G} *(\mathrm{C} 2 \mathrm{v})$

State $=2-\mathrm{A} 2 \backslash \mathrm{HF}=-116.4835863$

Potential energy minimum

single-point energy with the 6-311++G** basis: $\mathrm{HF}=-116.5152663$

$\begin{array}{lrr}0.000000 & 1.231166 & -0.197848 \\ 0.000000 & 0.000000 & 0.447178 \\ 0.000000 & -1.231166 & -0.197848 \\ 0.000000 & 2.151856 & 0.353754 \\ 0.000000 & 1.299089 & -1.270783 \\ 0.000000 & 0.000000 & 1.525159\end{array}$




\begin{tabular}{|c|c|c|c|}
\hline 1 & 0.000000 & -2.151856 & 0.353754 \\
\hline 1 & 0.000000 & -1.299089 & -1.270783 \\
\hline & $==========$ & $=========$ & $=====$ \\
\hline & $6-31 G(2 d f, p$ & (C1) (from & G4 calculation) \\
\hline & ------------- & -ーーーーーーーーーーー & ---- \\
\hline & $2-A \backslash E(U B 3 L Y$ & $=-117.27087$ & 2793 \\
\hline & ial energy & inimum & \\
\hline & rgy : -117.16 & 927 (enthapl) & $y:-117.160983)$ \\
\hline & $\mathrm{x}$ & $\mathrm{Y}$ & $\mathrm{Z}$ \\
\hline 6 & 1.227456 & -0.195280 & -0.000005 \\
\hline 6 & -0.000002 & 0.441011 & -0.000009 \\
\hline 1 & 2.156261 & 0.362017 & -0.000023 \\
\hline 1 & 1.296468 & -1.278356 & 0.000017 \\
\hline 1 & 0.000004 & 1.529986 & -0.000031 \\
\hline 6 & -1.227457 & -0.195281 & 0.000013 \\
\hline 1 & -1.296455 & -1.278358 & 0.000032 \\
\hline 1 & -2.156262 & 0.362014 & 0.000011 \\
\hline
\end{tabular}

\section{B3LYP/cC-pVTZ+d (C1) (from W1BD calculation)}

\begin{tabular}{|c|c|c|c|}
\hline \multicolumn{4}{|c|}{$\begin{array}{l}\text { State }=2-A \backslash E(R B 3 L Y P)=-117.309273065 \\
\text { potential energy minimum } \\
\text { W1BD-energy: }-117.211587 \text { (enthaply:-117.210643) }\end{array}$} \\
\hline & $\mathrm{X}$ & $\mathrm{Y}$ & $\mathrm{Z}$ \\
\hline & 1.224590 & -0.195297 & -0.000005 \\
\hline & -0.000001 & 0.440549 & -0.000009 \\
\hline & 2.149484 & 0.362340 & -0.000023 \\
\hline & 1.292866 & -1.275372 & 0.000016 \\
\hline & 0.000002 & 1.526337 & -0.000030 \\
\hline & -1.224590 & -0.195297 & 0.000013 \\
\hline & -1.292861 & -1.275373 & 0.000034 \\
\hline & -2.149485 & 0.362338 & 0.000009 \\
\hline
\end{tabular}

B 3LYP / 6-311++G** (C1)

$\begin{array}{rrrr}\text { State=2-A } \backslash \text { (UB3LYP }) & -117.298273923 \\ \text { Potential energy minimum } & \mathrm{Z} \\ 6 & 1.228102 & -0.196020 & -0.000003 \\ 6 & -0.000006 & 0.442224 & -0.000010 \\ 1 & 2.155196 & 0.362803 & -0.000020 \\ 1 & 1.296821 & -1.278536 & 0.000021 \\ 1 & 0.000011 & 1.530390 & -0.000034 \\ 6 & -1.228104 & -0.196023 & 0.000010 \\ 1 & -1.296784 & -1.278539 & 0.000028 \\ 1 & -2.155198 & 0.362797 & 0.000021\end{array}$

4. twisted allyl radical

$* * * * * * * * * * * * * * * * * * * * * * * *$

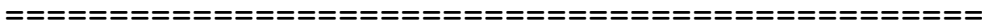
$\mathrm{UHF} / \mathrm{STO}-3 \mathrm{G}$ (Cs)

State $=2-A^{\prime} \backslash \mathrm{HF}=-115.0158088 \backslash \mathrm{S} 2=0.772562$

first oder saddle point

$$
\mathrm{X}
$$

$-1.238111$

0.000000

1.210823

$-1.481285$

$-2.080709$

0.192857
$\mathrm{Y}$

0.007877

0.440429

$-0.446581$

$-1.046068$

0.685771

1. 510710

\section{$\mathrm{Z}$}

0.000000

0.000000

0.000000

0.000000

0.000000

0.000000 


$\begin{array}{rrrr}1 & 1.766431 & -0.580382 & 0.921535 \\ 1 & 1.766431 & -0.580382 & -0.921535\end{array}$

\begin{tabular}{|c|c|c|c|}
\hline \multicolumn{4}{|c|}{$\begin{array}{l}\text { State }=2-A^{\prime} \backslash H F=-115.0124915 \\
\text { first oder saddle point }\end{array}$} \\
\hline & $\mathrm{X}$ & $\mathrm{Y}$ & $\mathrm{Z}$ \\
\hline 6 & -1.239198 & 0.009941 & 0.000000 \\
\hline 6 & 0.000000 & 0.435283 & 0.000000 \\
\hline 6 & 1.205916 & -0.459629 & 0.000000 \\
\hline 1 & -1.488077 & -1.042544 & 0.000000 \\
\hline 1 & -2.078136 & 0.692191 & 0.000000 \\
\hline 1 & 0.199692 & 1.504023 & 0.000000 \\
\hline 1 & 1.783108 & -0.533620 & 0.915701 \\
\hline 1 & 1.783108 & -0.533620 & -0.915701 \\
\hline
\end{tabular}

State $=2-\mathrm{A}^{\prime} \backslash \mathrm{HF}=-115.0547881$

first oder saddle point

\begin{tabular}{lrrr} 
& \multicolumn{1}{c}{$\mathrm{X}$} & \multicolumn{1}{c}{$\mathrm{Y}$} & $\mathrm{Z}$ \\
6 & -1.257609 & -0.032537 & 0.000000 \\
6 & 0.000000 & 0.443559 & 0.000000 \\
6 & 1.226611 & -0.423355 & 0.000000 \\
1 & -1.468329 & -1.092879 & 0.000000 \\
1 & -2.113872 & 0.627229 & 0.000000 \\
1 & 0.161625 & 1.517819 & 0.000000 \\
1 & 1.803282 & -0.489086 & 0.916142 \\
1 & 1.803282 & -0.489086 & -0.916142
\end{tabular}

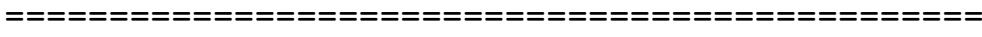
$\mathrm{UHF} / 3-21 \mathrm{G}$ (Cs)

$\begin{array}{lrrr}\text { State }=2-A^{\prime} \backslash H F=-115.7927605 \backslash S 2=0.86194 \\ \text { first oder saddle point } & \mathrm{Z} \\ 6 & -1.233656 & -0.034272 & 0.000000 \\ 6 & 0.000000 & 0.452875 & 0.000000 \\ 6 & 1.223122 & -0.382808 & 0.000000 \\ 1 & -1.422900 & -1.091096 & 0.000000 \\ 1 & -2.092366 & 0.609665 & 0.000000 \\ 1 & 0.142730 & 1.522242 & 0.000000 \\ 1 & 1.717867 & -0.627790 & 0.922143 \\ 1 & 1.717867 & -0.627790 & -0.922143\end{array}$

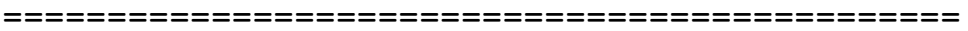
ROHF/3-21G (Cs)

\begin{tabular}{lrrr} 
State=2-A $\backslash$ HF $=-115.7887007$ \\
first oder saddle point \\
\multicolumn{1}{c}{$\mathrm{X}$} & $\mathrm{Y}$ & $\mathrm{Z}$ \\
6 & -1.232284 & -0.019353 & 0.000000 \\
6 & 0.000000 & 0.445312 & 0.000000 \\
6 & 1.211968 & -0.408975 & 0.000000 \\
1 & -1.438724 & -1.072865 & 0.000000 \\
1 & -2.081408 & 0.637140 & 0.000000 \\
1 & 0.162822 & 1.511379 & 0.000000 \\
1 & 1.739604 & -0.588778 & 0.917909 \\
1 & 1.739604 & -0.588778 & -0.917909
\end{tabular}




\begin{tabular}{|c|c|c|c|}
\hline \multicolumn{4}{|c|}{$\operatorname{CAS}(2,3) / 3-21 \mathrm{G} \quad(\mathrm{Cs})$} \\
\hline \multicolumn{4}{|c|}{$\begin{array}{l}\text { State }=2-A^{\prime} \backslash H F=-115.8183881 \\
\text { first oder saddle point }\end{array}$} \\
\hline & $\mathrm{x}$ & $\mathrm{Y}$ & $\mathrm{Z}$ \\
\hline 6 & -1.242633 & -0.044671 & 0.000000 \\
\hline 6 & 0.000000 & 0.452294 & 0.000000 \\
\hline 6 & 1.224073 & -0.387070 & 0.000000 \\
\hline 1 & -1.425469 & -1.102468 & 0.000000 \\
\hline 1 & -2.103392 & 0.596613 & 0.000000 \\
\hline 1 & 0.141497 & 1.521073 & 0.000000 \\
\hline 1 & 1.749362 & -0.569265 & 0.918257 \\
\hline 1 & 1.749362 & -0.569265 & -0.918257 \\
\hline
\end{tabular}

\section{$\mathrm{UHF} / 6-31 \mathrm{G} *$ (Cs)}

State $=2-A^{\prime} \backslash \mathrm{HF}=-116.4385098 \backslash \mathrm{S} 2=0.83542$

first oder saddle point

$\mathrm{X}$

$\mathrm{Y}$

$\mathrm{Z}$

$\begin{array}{rrrr}6 & -1.238369 & -0.031789 & 0.000000 \\ 6 & 0.000000 & 0.443770 & 0.000000 \\ 6 & 1.223016 & -0.387791 & 0.000000 \\ 1 & -1.440183 & -1.088811 & 0.000000 \\ 1 & -2.090575 & 0.624515 & 0.000000 \\ 1 & 0.140910 & 1.516328 & 0.000000 \\ 1 & 1.740982 & -0.598585 & 0.919608 \\ 1 & 1.740982 & -0.598585 & -0.919608\end{array}$

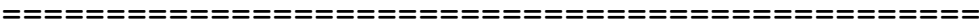
$\mathrm{ROHF} / 6-31 \mathrm{G} *$ ( Cs )

State $=2-A^{\prime} \backslash \mathrm{HF}=-116.4338996$

first oder saddle point

$\mathrm{X}$

$\mathrm{Y}$

$\mathrm{Z}$

$\begin{array}{rrrr}6 & -1.237754 & -0.020461 & 0.000000 \\ 6 & 0.000000 & 0.436302 & 0.000000 \\ 6 & 1.213199 & -0.411825 & 0.000000 \\ 1 & -1.453660 & -1.074696 & 0.000000 \\ 1 & -2.081672 & 0.646500 & 0.000000 \\ 1 & 0.157554 & 1.506298 & 0.000000 \\ 1 & 1.762555 & -0.551099 & 0.914451 \\ 1 & 1.762555 & -0.551099 & -0.914451\end{array}$

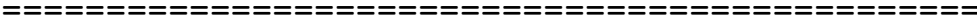
$\operatorname{CAS}(2,3) / 6-31 \mathrm{G} *(\mathrm{Cs})$

State $=2-A^{\prime} \backslash \mathrm{HF}=-116.4621532$

first oder saddle point

single-point energy with the 6-311++G** basis: $\mathrm{HF}=-116.493977$

$\mathrm{X}$

$$
\begin{array}{r}
-1.248495 \\
0.000000 \\
1.225452 \\
-1.442061 \\
-2.104100
\end{array}
$$

$\mathrm{Y}$

$$
\begin{array}{r}
-0.046119 \\
0.442269 \\
-0.388959 \\
-1.104306 \\
0.605368
\end{array}
$$

Z
0.000000
0.000000
0.000000
0.000000
0.000000 


\begin{tabular}{|c|c|c|c|}
\hline$\perp$ & 0.136752 & 1.514639 & 0.000000 \\
\hline 1 & 1.773833 & -0.529423 & 0.914628 \\
\hline$\perp$ & 1.773833 & -0.529423 & -0.914628 \\
\hline \multicolumn{4}{|c|}{ 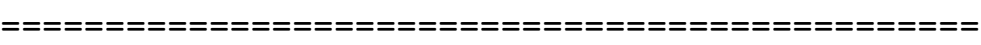 } \\
\hline \multicolumn{4}{|c|}{ B3LYP/6-31G(2df,p) (C1) (from G4 calculation) } \\
\hline \multirow{2}{*}{\multicolumn{4}{|c|}{$\begin{array}{l}\text { State }=2-\mathrm{A} \backslash \mathrm{E}(\mathrm{UB} 3 \mathrm{LYP})=-117.243240828 \\
\text { First order saddle point }\end{array}$}} \\
\hline & & & \\
\hline \multicolumn{4}{|c|}{ G4-energy: -117.140014 (enthaply:-117.139070) } \\
\hline & $\mathrm{x}$ & $\mathrm{Y}$ & $\mathrm{z}$ \\
\hline 6 & 1.220224 & -0.237154 & 0.000000 \\
\hline 6 & 0.067822 & 0.430826 & -0.000001 \\
\hline 1 & 2.174350 & 0.279840 & 0.000000 \\
\hline 1 & 1.251363 & -1.322287 & 0.000000 \\
\hline 1 & 0.109543 & 1.525160 & 0.000000 \\
\hline 6 & -1.271622 & -0.167576 & 0.000000 \\
\hline 1 & -1.816902 & -0.319648 & -0.926464 \\
\hline & -1.816898 & -0.319644 & 0.926466 \\
\hline \multicolumn{4}{|c|}{ =" =" =" =" } \\
\hline \multicolumn{4}{|c|}{ B3LYP/cC-pVTZ+d (C1) (from W1BD calculation) } \\
\hline \multirow{2}{*}{\multicolumn{4}{|c|}{$\begin{array}{l}\text { State }=2-\mathrm{A} \backslash \mathrm{E}(\mathrm{UB} 3 \mathrm{LYP})=-117.281892941 \\
\text { First order saddle point }\end{array}$}} \\
\hline & & & \\
\hline \multicolumn{4}{|c|}{ W1BD-energy: -117.188108 (enthaply: -117.187164 ) } \\
\hline & $\mathrm{x}$ & $\mathrm{Y}$ & $\mathrm{z}$ \\
\hline 6 & 1.216932 & -0.237989 & 0.000000 \\
\hline 6 & 0.068091 & 0.430337 & 0.000000 \\
\hline 1 & 2.166524 & 0.280650 & 0.000001 \\
\hline 1 & 1.245021 & -1.320230 & -0.000004 \\
\hline 1 & 0.113605 & 1.521273 & 0.000003 \\
\hline 6 & -1.269686 & -0.165060 & 0.000000 \\
\hline 1 & -1.808592 & -0.322703 & -0.925189 \\
\hline 1 & -1.808586 & -0.322715 & 0.925190 \\
\hline \multirow{2}{*}{\multicolumn{4}{|c|}{$\begin{array}{l}============================================ \\
\text { B3LYP / } 6-311++G * \text { ( C } 1 \text { ) }\end{array}$}} \\
\hline & & & \\
\hline \multirow{2}{*}{\multicolumn{4}{|c|}{$\begin{array}{l}\text { State }=2-\mathrm{A} \backslash \mathrm{E}(\mathrm{UB} 3 \mathrm{LYP})=-117.271134362 \\
\text { First order saddle point }\end{array}$}} \\
\hline & & & \\
\hline & $\mathrm{x}$ & $\mathrm{Y}$ & $\mathrm{Z}$ \\
\hline 6 & 1.220329 & -0.238854 & 0.000045 \\
\hline 6 & 0.067785 & 0.432355 & -0.000114 \\
\hline 1 & 2.172189 & 0.280765 & -0.000026 \\
\hline 1 & 1.247859 & -1.323539 & 0.000289 \\
\hline 1 & 0.112323 & 1.525695 & -0.000183 \\
\hline 6 & -1.272919 & -0.165487 & 0.000016 \\
\hline 1 & -1.811766 & -0.326108 & -0.927696 \\
\hline 1 & -1.811772 & -0.324896 & 0.927938 \\
\hline
\end{tabular}

5. planar allyl anion

$* * * * * * * * * * * * * * * * * * * * *$

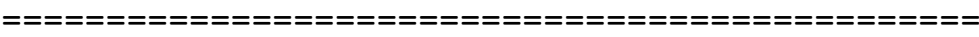
$\mathrm{HF} / \mathrm{STO}-3 \mathrm{G} \quad(\mathrm{C} 2 \mathrm{v})$

State $=1-\mathrm{A} 1 \backslash \mathrm{HF}=-114.8384175$

Potential energy minimum

$\mathrm{x}$ 0.000000

0.000000

0.000000

0.000000

0.000000
$\mathrm{Y}$

1.253672

0.000000

$-1.253672$

2.132612

1.415590
$-0.176125$

0.373580

$-0.176125$

0.438564

$-1.236439$ 


$\begin{array}{rrrr}1 & 0.000000 & 0.000000 & 1.467776 \\ 1 & 0.000000 & -2.132612 & 0.438564 \\ 1 & 0.000000 & -1.415590 & -1.236439\end{array}$

\begin{tabular}{|c|c|c|c|}
\hline \multicolumn{4}{|c|}{$\operatorname{CAS}(2,3) / \mathrm{STO}-3 \mathrm{G} \quad(\mathrm{C} 2 \mathrm{v})$} \\
\hline \multicolumn{4}{|c|}{$\begin{array}{l}\text { State }=1-\mathrm{A} 1 \backslash \mathrm{HF}=-114.8710059 \\
\text { second order saddle point }\end{array}$} \\
\hline & $\mathrm{X}$ & $\mathrm{Y}$ & $\mathrm{Z}$ \\
\hline 6 & 0.000000 & 1.260189 & -0.178950 \\
\hline 6 & 0.000000 & 0.000000 & 0.382954 \\
\hline 6 & 0.000000 & -1.260189 & -0.178950 \\
\hline 1 & 0.000000 & 2.145492 & 0.428943 \\
\hline 1 & 0.000000 & 1.419686 & -1.241118 \\
\hline 1 & 0.000000 & 0.000000 & 1.474019 \\
\hline 1 & 0.000000 & -2.145492 & 0.428943 \\
\hline 1 & 0.000000 & -1.419686 & -1.241118 \\
\hline \multicolumn{4}{|c|}{ 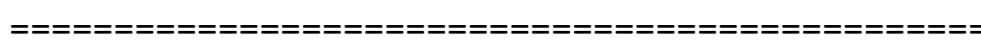 } \\
\hline \multirow{2}{*}{\multicolumn{4}{|c|}{ CAS $(2,3) /$ STO-3G (no symmetry, pyramidalized) }} \\
\hline & & & \\
\hline \multicolumn{4}{|c|}{$\begin{array}{l}\text { State }=1-\mathrm{A} 1 \backslash \mathrm{HF}=-114.8710059 \\
\text { second order saddle point }\end{array}$} \\
\hline & $\mathrm{x}$ & $\mathrm{Y}$ & Z \\
\hline 6 & 0.000000 & 1.260189 & -0.178950 \\
\hline 6 & 0.000000 & 0.000000 & 0.382954 \\
\hline 6 & 0.000000 & -1.260189 & -0.178950 \\
\hline 1 & 0.000000 & 2.145492 & 0.428943 \\
\hline 1 & 0.000000 & 1.419686 & -1.241118 \\
\hline 1 & 0.000000 & 0.000000 & 1.474019 \\
\hline 1 & 0.000000 & -2.145492 & 0.428943 \\
\hline 1 & 0.000000 & -1.419686 & -1.241118 \\
\hline
\end{tabular}

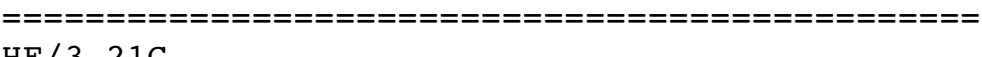
$\mathrm{HF} / 3-21 \mathrm{G}$

State $=1-\mathrm{A} 1 \backslash \mathrm{HF}=-115.7461332$

Potential energy minimum $\mathrm{x}$ 0.000000 0.000000

0.000000

0.000000

0.000000

0.000000

0.000000

0.000000
$\mathrm{Y}$

1.264876

0.000000

$-1.264876$

2.145093

1.420910

0.000000

$-2.145093$

$-1.420910$ check!
$-0.176930$

0.376003

$-0.176930$

0.444783

$-1.243945$

1. 465461

0.444783

$-1.243945$
State $=1-\mathrm{A} 1 \backslash \mathrm{HF}=-115.7673846$

Potential energy minimum

$\mathrm{X}$
0.000000
0.000000
0.000000
0.000000
0.000000
0.000000
0.000000

$\mathrm{Y}$

$\begin{array}{rr}1.269617 & -0.178879 \\ 0.000000 & 0.381809 \\ -1.269617 & -0.178879 \\ 2.152890 & 0.439463 \\ 1.423350 & -1.247124 \\ 0.000000 & 1.471017 \\ -2.152890 & 0.439463\end{array}$




$\begin{array}{rrrr}6 & 0.000000 & 1.270573 & -0.174928 \\ 6 & 0.000000 & 0.000000 & 0.368167 \\ 6 & 0.000000 & -1.270573 & -0.174928 \\ 1 & 0.000000 & 2.145046 & 0.458352 \\ 1 & 0.000000 & 1.440139 & -1.243101 \\ 1 & 0.000000 & 0.000000 & 1.459637 \\ 1 & 0.000000 & -2.145046 & 0.458352 \\ 1 & 0.000000 & -1.440139 & -1.243101\end{array}$

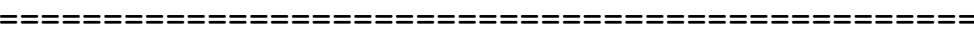
$\operatorname{CAS}(2,3) / 6-31 \mathrm{G} *(\mathrm{C} 2 \mathrm{v})$

State $=1-\mathrm{A} 1 \backslash \mathrm{HF}=-116.4133991$

second order saddle point

single-point energy with the $6-311++G * *$ basis: $\mathrm{HF}=-116.2511931$

$\begin{array}{rrrr}6 & 0.000000 & 1.274965 & -0.176753 \\ 6 & 0.000000 & 0.000000 & 0.373581 \\ 6 & 0.000000 & -1.274965 & -0.176753 \\ 1 & 0.000000 & 2.152404 & 0.452797 \\ 1 & 0.000000 & 1.443517 & -1.245554 \\ 1 & 0.000000 & 0.00000 & 1.465061 \\ 1 & 0.000000 & -2.152404 & 0.452797 \\ 1 & 0.000000 & -1.443517 & -1.245554\end{array}$

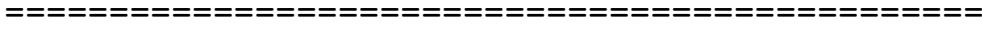
$\operatorname{CAS}(2,3) / 6-31 G *(C 2$, pyramidalized $)$

State $=1-\mathrm{A} \backslash \mathrm{HF}=-116.4140757$ potential energy minimum single-point energy with the $6-311++G * *$ basis: $\mathrm{HF}=-116.4713863$

$\mathrm{X}$ $\mathrm{Y} \quad \mathrm{Z}$

$\begin{array}{lrrr}6 & 0.000000 & 1.275468 & -0.176745 \\ 6 & 0.000000 & 0.000000 & 0.379202 \\ 6 & 0.000000 & -1.275468 & -0.176745 \\ 1 & -0.284940 & 2.124577 & 0.432627 \\ 1 & -0.130644 & 1.409667 & -1.245064 \\ 1 & 0.000000 & 0.000000 & 1.470599 \\ 1 & 0.284940 & -2.124577 & 0.432627 \\ 1 & 0.130644 & -1.409667 & -1.245064 \\ ======================================= \\ \text { B3LYP } / 6-31 \mathrm{G}(2 \mathrm{df}, \mathrm{p}) & (\mathrm{C} 1) & \text { (from G4 } \text { calculation) }\end{array}$

State $=1-A \backslash E($ RB3LYP $)=-117.254667596$

Potential energy minimum

G4-energy:-117.180369 (enthaply:-117.179425)

$\begin{array}{cccr} & \mathrm{X} & \mathrm{Y} & \mathrm{Z} \\ 6 & 1.277973 & -0.174987 & 0.000013 \\ 6 & 0.000000 & 0.368816 & -0.000002 \\ 1 & 2.160418 & 0.458929 & -0.000026\end{array}$




$\begin{array}{rrrr}1 & 1.449698 & -1.251170 & 0.000024 \\ 1 & 0.000000 & 1.471423 & -0.000025 \\ 6 & -1.277973 & -0.174987 & -0.000020 \\ 1 & -1.449698 & -1.251170 & 0.000038 \\ 1 & -2.160418 & 0.458929 & 0.000041\end{array}$

$=========================================$ B3LYP/cc-pVTZ+d (C1) (from W1BD calculation)

State $=1-A \backslash E($ RB3LYP $)=-117.314160692$

Potential energy minimum

W1BD-energy:-117.229109 (enthaply:-117.228164)

\begin{tabular}{rrrr} 
& $\mathrm{X}$ & \multicolumn{1}{c}{$\mathrm{Y}$} & $\mathrm{Z}$ \\
6 & 1.274346 & -0.177467 & 0.000005 \\
6 & 0.000000 & 0.375889 & -0.000004 \\
1 & 2.155906 & 0.452834 & -0.000024 \\
1 & 1.436114 & -1.252276 & 0.000020 \\
1 & 0.000000 & 1.473154 & -0.000025 \\
6 & -1.274346 & -0.177467 & -0.000007 \\
1 & -1.436113 & -1.252276 & 0.000037 \\
1 & -2.155905 & 0.452834 & 0.000029
\end{tabular}

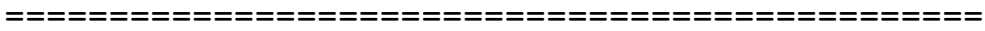
B3LYP / 6-311++G** (C1)

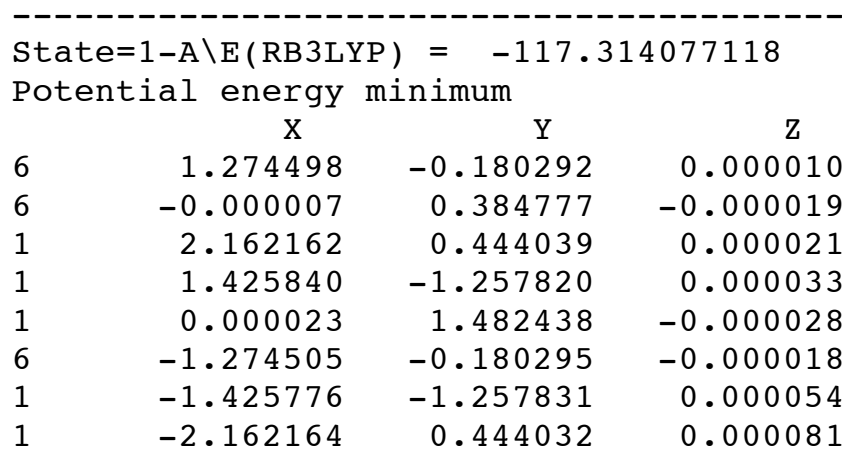

6. twisted allyl anion

$* * * * * * * * * * * * * * * * * * * * * *$

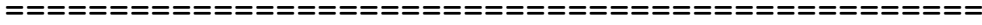
$\mathrm{HF} / \mathrm{STO}-3 \mathrm{G}$

\begin{tabular}{|c|c|c|c|}
\hline \multicolumn{4}{|c|}{$\begin{array}{l}\text { State }=1-A^{\prime} \backslash H F=-114.7981644 \\
\text { first oder saddle point }\end{array}$} \\
\hline & $\mathrm{x}$ & $\mathrm{Y}$ & $\mathrm{Z}$ \\
\hline 6 & -1.262818 & 0.003752 & 0.000000 \\
\hline 6 & 0.000000 & 0.385099 & 0.000000 \\
\hline & 1.244215 & -0.540828 & 0.000000 \\
\hline 1 & -1.545580 & -1.037699 & 0.000000 \\
\hline 1 & -2.096173 & 0.694544 & 0.000000 \\
\hline 1 & 0.114594 & 1.484086 & 0.000000 \\
\hline 1 & 1.819387 & -0.114536 & 0.852761 \\
\hline 1 & 1.819387 & -0.114536 & -0.852761 \\
\hline \multicolumn{4}{|c|}{ 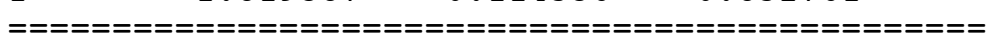 } \\
\hline \multicolumn{4}{|c|}{$\operatorname{CAS}(2,3) / \mathrm{STO}-3 \mathrm{G}$} \\
\hline \multicolumn{4}{|c|}{$\begin{array}{l}\text { State }=1-\mathrm{A} 1 \backslash \mathrm{HF}=-114.8447245 \\
\text { first oder saddle point }\end{array}$} \\
\hline & $\mathrm{x}$ & $\mathrm{Y}$ & $\mathrm{Z}$ \\
\hline 6 & -1.275998 & -0.039373 & 0.000000 \\
\hline 6 & 0.000000 & 0.398138 & 0.000000 \\
\hline & 1.262143 & -0.503777 & 0.000000 \\
\hline & -1.517943 & -1.092484 & 0.000000 \\
\hline
\end{tabular}




$\begin{array}{rrrr}1 & -2.136294 & 0.620044 & 0.000000 \\ 1 & 0.067559 & 1.499109 & 0.000000 \\ 1 & 1.834903 & -0.078299 & 0.854144 \\ 1 & 1.834903 & -0.078299 & -0.854144\end{array}$

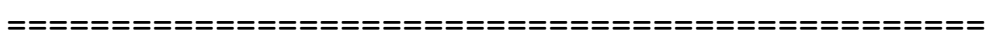
$\mathrm{HF} / 3-21 \mathrm{G}$

State $=1-\mathrm{A}^{\prime} \backslash \mathrm{HF}=-115.7064361 \backslash$

first oder saddle point

\begin{tabular}{rrrr} 
& \multicolumn{1}{c}{$\mathrm{X}$} & \multicolumn{1}{c}{$\mathrm{Y}$} & $\mathrm{Z}$ \\
6 & -1.257879 & -0.019098 & 0.000000 \\
6 & 0.000000 & 0.405647 & 0.000000 \\
6 & 1.223641 & -0.504444 & 0.000000 \\
1 & -1.462211 & -1.074811 & 0.000000 \\
1 & -2.107322 & 0.650760 & 0.000000 \\
1 & 0.098621 & 1.503522 & 0.000000 \\
1 & 1.838171 & -0.186051 & 0.871976 \\
1 & 1.838171 & -0.186051 & -0.871976
\end{tabular}

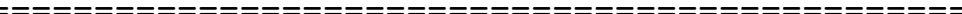
$\operatorname{CAS}(2,3) / 3-21 \mathrm{G}$

State $=1-A^{\prime} \backslash \mathrm{HF}=-115.7334726$

first oder saddle point

$\mathrm{X}$ $\mathrm{Y}$

$$
\begin{array}{r}
-0.041744 \\
0.416214 \\
-0.486375 \\
-1.102414 \\
0.609032 \\
1.515816 \\
-0.175503 \\
-0.175503
\end{array}
$$

\section{$\mathrm{Z}$}

$$
\begin{array}{r}
0.000000 \\
0.0000000 \\
0.000000 \\
0.000000 \\
0.000000 \\
0.000000 \\
0.873670 \\
-0.873670
\end{array}
$$

\section{$\mathrm{HF} / 6-31 \mathrm{G}$ *}

\section{State $=1-A^{\prime} \backslash \mathrm{HF}=-116.1389889$}

first oder saddle point

$$
\mathrm{X}
$$

$$
\begin{array}{r}
-1.259192 \\
0.000000 \\
1.231823 \\
-1.469543 \\
-2.109585 \\
0.070811 \\
1.836265 \\
1.836265
\end{array}
$$

$\mathrm{Y}$

$\mathrm{Z}$

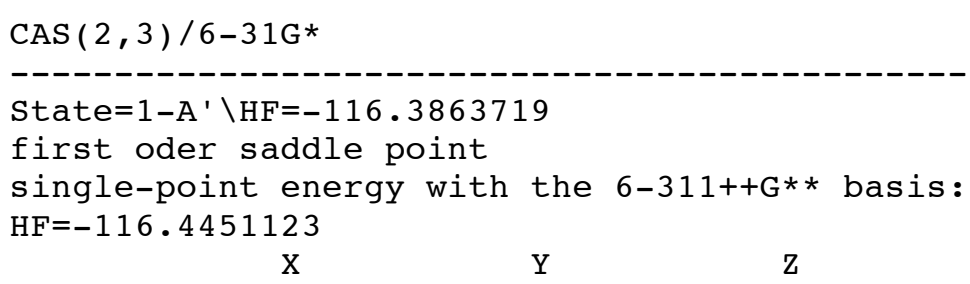

$\begin{array}{rrrr}6 & -1.266445 & -0.063318 & 0.000000 \\ 6 & 0.000000 & 0.399970 & 0.000000 \\ 6 & 1.240423 & -0.461824 & 0.000000\end{array}$




$\begin{array}{rrrr}1 & -1.454139 & -1.125619 & 0.000000 \\ 1 & -2.131580 & 0.590362 & 0.000000 \\ 1 & 0.049853 & 1.503666 & 0.000000 \\ 1 & 1.845999 & -0.108686 & 0.863217 \\ 1 & 1.845999 & -0.108686 & -0.863217\end{array}$

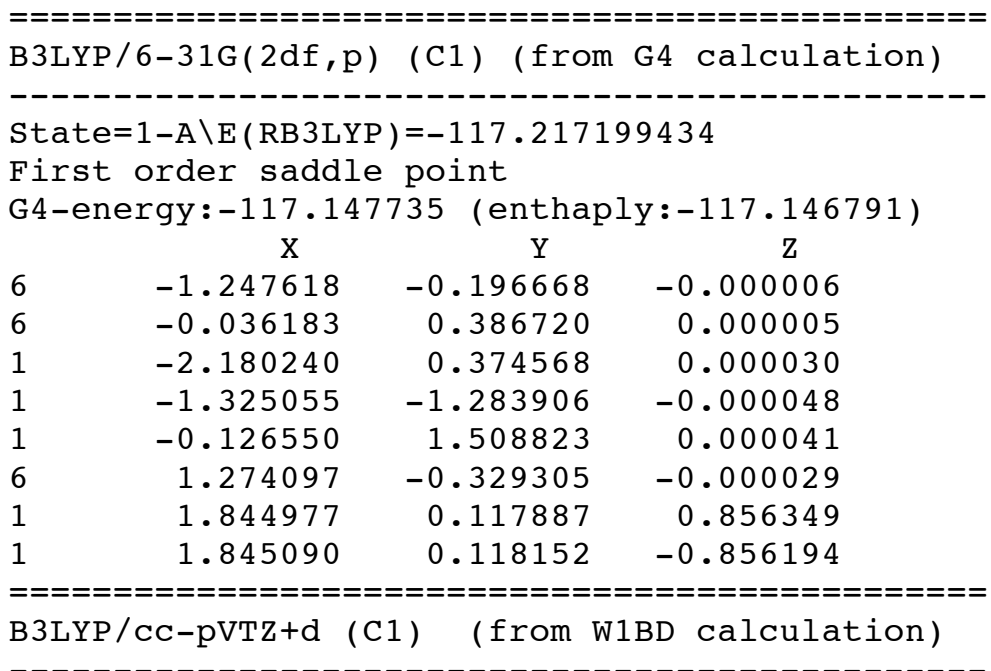

State $=1-A \backslash E($ RB3LYP $)=-117.277370158$

First order saddle point

W1BD-energy:-117.197553 (enthaply:-117.196609)

$$
\mathrm{X} \quad \mathrm{Y} \text { Z }
$$

$\begin{array}{rrrr}6 & -1.245569 & -0.200090 & -0.000001 \\ 6 & -0.039984 & 0.387367 & 0.000001 \\ 1 & -2.170720 & 0.377564 & 0.000005 \\ 1 & -1.317027 & -1.284792 & -0.000009 \\ 1 & -0.117657 & 1.503810 & 0.000008 \\ 6 & 1.269995 & -0.311643 & -0.000005 \\ 1 & 1.849369 & 0.074785 & 0.864904 \\ 1 & 1.849387 & 0.074832 & -0.864880\end{array}$

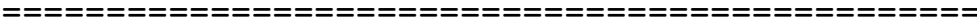
B3LYP / 6-311++G** (C1)

State $=1-\mathrm{A} \backslash \mathrm{E}(\mathrm{RB} 3 \mathrm{LYP})=-117.278825880$

First order saddle point

\begin{tabular}{lrrr} 
& $\mathrm{X}$ & \multicolumn{1}{c}{$\mathrm{Y}$} & $\mathrm{Z}$ \\
6 & 1.247497 & -0.206369 & 0.000056 \\
6 & 0.043600 & 0.393019 & -0.000094 \\
1 & 2.174922 & 0.370834 & -0.000118 \\
1 & 1.315996 & -1.292641 & 0.000353 \\
1 & 0.117172 & 1.509254 & -0.000337 \\
6 & -1.271833 & -0.287912 & 0.000160 \\
1 & -1.861554 & 0.009168 & -0.884188 \\
1 & -1.862123 & 0.010958 & 0.883564
\end{tabular}

7. planar benzyl cation

$* * * * * * * * * * * * * * * * * * * * * * *$

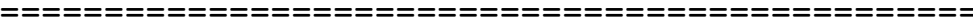
$\operatorname{CAS}(4,6) / 3-21 \mathrm{G}$

State $=1-\mathrm{A} 1 \backslash \mathrm{HF}=-267.4307213$

potential energy minimum

$\begin{array}{rccr} & X & Y & Z \\ 6 & 0.000000 & 0.000000 & 0.983925 \\ 6 & 0.000000 & 0.000000 & -1.796970\end{array}$




$\begin{array}{rrrr}6 & 0.000000 & 1.237575 & 0.255481 \\ 6 & 0.000000 & -1.237575 & 0.255481 \\ 6 & 0.000000 & -1.228053 & -1.116602 \\ 6 & 0.000000 & 1.228053 & -1.116602 \\ 1 & 0.000000 & 2.162470 & 0.796975 \\ 1 & 0.000000 & -2.162470 & 0.796975 \\ 1 & 0.000000 & -2.143505 & -1.670108 \\ 1 & 0.000000 & 2.143505 & -1.670108 \\ 1 & 0.000000 & 0.000000 & -2.868963 \\ 6 & 0.000000 & 0.000000 & 2.337646 \\ 1 & 0.000000 & -0.914020 & 2.900538 \\ 1 & 0.000000 & 0.914020 & 2.900538\end{array}$

\begin{tabular}{|c|c|c|c|}
\hline \multicolumn{4}{|c|}{$G(2 d f, p)$} \\
\hline \multicolumn{4}{|c|}{$\begin{array}{l}\text { State }=1-\mathrm{A} 1 \backslash \mathrm{HF}=-270.6812673 \\
\text { potential energy minimum } \\
\mathrm{G} 4 \text {-energy: }-270.460553 \text { (enthaply: }-270.459608)\end{array}$} \\
\hline & $\mathrm{X}$ & $\mathrm{Y}$ & $\mathrm{Z}$ \\
\hline 6 & 0.000000 & 0.000000 & 0.980416 \\
\hline 6 & 0.000000 & 0.000000 & -1.797783 \\
\hline 6 & 0.000000 & 1.243961 & 0.253624 \\
\hline 6 & 0.000000 & -1.243961 & 0.253624 \\
\hline 6 & 0.000000 & -1.233603 & -1.118712 \\
\hline 6 & 0.000000 & 1.233603 & -1.118712 \\
\hline 1 & 0.000000 & 2.176188 & 0.808534 \\
\hline 1 & 0.000000 & -2.176188 & 0.808534 \\
\hline 1 & 0.000000 & -2.159288 & -1.681415 \\
\hline 1 & 0.000000 & 2.159288 & -1.681415 \\
\hline 1 & 0.000000 & 0.000000 & -2.883861 \\
\hline 6 & 0.000000 & 0.000000 & 2.347226 \\
\hline 1 & 0.000000 & -0.925990 & 2.915756 \\
\hline 1 & 0.000000 & 0.925990 & 2.915756 \\
\hline & $========$ & $=========$ & $====$ \\
\hline & $11++G * *$ & & \\
\hline
\end{tabular}

State $=1-A^{\prime} \backslash E($ RB3LYP $)=-270.725073707$

Potential energy minimum $\mathrm{X}$

$\begin{array}{rrrr}6 & 0.000014 & 0.981579 & 0.000000 \\ 6 & 0.000030 & 0.253588 & 1.244197 \\ 6 & 0.000030 & -1.119187 & 1.233294 \\ 6 & -0.000030 & -1.799109 & 0.000000 \\ 6 & 0.000030 & -1.119187 & -1.233294 \\ 6 & 0.000030 & 0.253588 & -1.244197 \\ 1 & 0.000030 & 0.805159 & 2.177557 \\ 1 & 0.000068 & -1.681045 & 2.158748 \\ 1 & -0.000167 & -2.884252 & 0.000000 \\ 1 & 0.000068 & -1.681045 & -2.158748 \\ 1 & 0.000030 & 0.805159 & -2.177557 \\ 6 & -0.000074 & 2.349334 & 0.000000 \\ 1 & -0.000103 & 2.916192 & 0.925906 \\ 1 & -0.000103 & 2.916192 & -0.925906\end{array}$

8. twisted benzyl cation

$* * * * * * * * * * * * * * * * * * * * * * * *$

State $=1-\mathrm{A} 1 \backslash \mathrm{HF}=-267.3495271$

first order saddle point 


\begin{tabular}{lrrr} 
& $\mathrm{X}$ & \multicolumn{1}{c}{$\mathrm{Y}$} & $\mathrm{Z}$ \\
6 & 0.000000 & 0.944906 & 0.000000 \\
6 & 0.000000 & -1.814662 & 0.000000 \\
6 & 0.000000 & 0.260806 & 1.223599 \\
6 & 0.000000 & 0.260806 & -1.223599 \\
6 & 0.000000 & -1.129096 & -1.200365 \\
6 & 0.000000 & -1.129096 & 1.200365 \\
1 & 0.000000 & 0.784723 & 2.158586 \\
1 & 0.000000 & 0.784723 & -2.158586 \\
1 & 0.000000 & -1.662149 & -2.128775 \\
1 & 0.000000 & -1.662149 & 2.128775 \\
1 & 0.000000 & -2.885102 & 0.000000 \\
6 & 0.000000 & 2.394926 & 0.000000 \\
1 & -0.923215 & 2.954331 & 0.000000 \\
1 & 0.923215 & 2.954331 & 0.000000
\end{tabular}

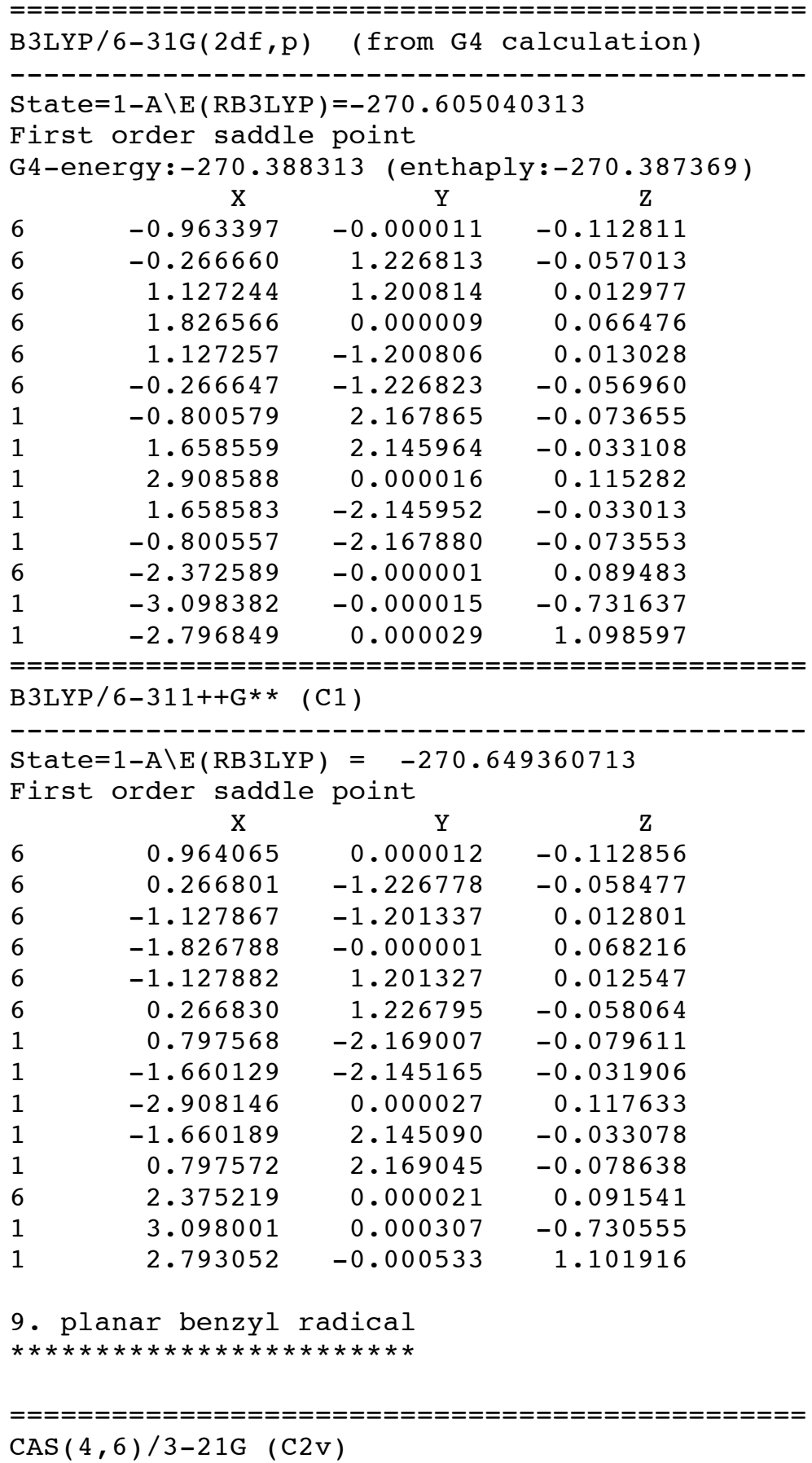




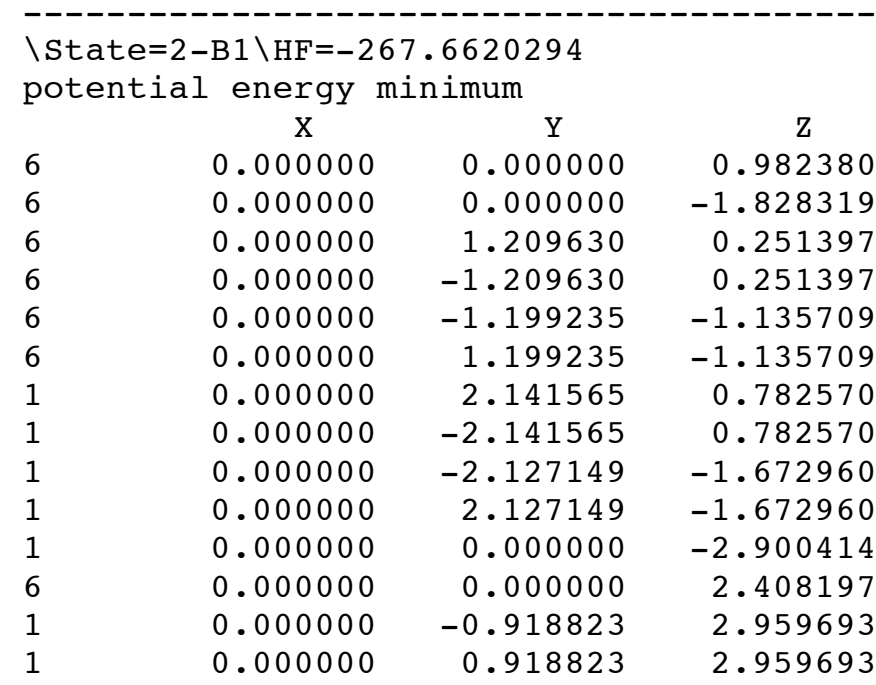

\begin{tabular}{|c|c|c|c|}
\hline \multicolumn{4}{|c|}{ B3LYP / 6-31G $(2 d f, p)$} \\
\hline \multicolumn{4}{|c|}{$\begin{array}{l}\text { State }=2-B 1 \backslash \mathrm{HF}=-270.9341523 \mathrm{G} \\
\text { potential energy minimum } \\
\text { G4-energy: }-270.728922 \text { (enthaply:-270.727978) }\end{array}$} \\
\hline & $\mathrm{x}$ & $\mathrm{Y}$ & $\mathrm{z}$ \\
\hline 6 & 0.000000 & 0.000000 & 0.993480 \\
\hline 6 & 0.000000 & 0.000000 & -1.835697 \\
\hline 6 & 0.000000 & 1.215556 & 0.251266 \\
\hline 6 & 0.000000 & -1.215556 & 0.251266 \\
\hline 6 & 0.000000 & -1.209244 & -1.131119 \\
\hline 6 & 0.000000 & 1.209244 & -1.131119 \\
\hline 1 & 0.000000 & 2.156346 & 0.793175 \\
\hline 1 & 0.000000 & -2.156346 & 0.793175 \\
\hline 1 & 0.000000 & -2.149485 & -1.673329 \\
\hline 1 & 0.000000 & 2.149485 & -1.673329 \\
\hline 1 & 0.000000 & 0.000000 & -2.920167 \\
\hline 6 & 0.000000 & 0.000000 & 2.396267 \\
\hline 1 & 0.000000 & -0.927082 & 2.957213 \\
\hline 1 & 0.000000 & 0.927082 & 2.957213 \\
\hline \multicolumn{4}{|c|}{ 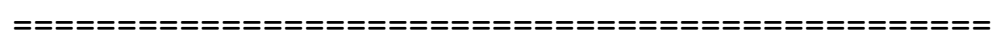 } \\
\hline \multicolumn{4}{|c|}{ B $3 L Y P / 6-311++G * * \quad(C s)$} \\
\hline \multicolumn{4}{|c|}{$\begin{array}{l}\text { State }=2-A^{\prime} \backslash E(U B 3 L Y P)=-270.987311951 \\
\text { Potential energy minimum }\end{array}$} \\
\hline & $\mathrm{x}$ & $\mathrm{Y}$ & $\mathrm{Z}$ \\
\hline 6 & -0.000194 & 0.994266 & 0.000000 \\
\hline 6 & 0.000100 & 0.251577 & 1.216887 \\
\hline 6 & 0.000100 & -1.132022 & 1.210342 \\
\hline 6 & 0.000041 & -1.837104 & 0.000000 \\
\hline 6 & 0.000100 & -1.132022 & -1.210342 \\
\hline 6 & 0.000100 & 0.251577 & -1.216887 \\
\hline 1 & 0.000233 & 0.790927 & 2.158163 \\
\hline 1 & 0.000177 & -1.674216 & 2.149609 \\
\hline 1 & -0.000011 & -2.920805 & 0.000000 \\
\hline 1 & 0.000177 & -1.674216 & -2.149609 \\
\hline 1 & 0.000233 & 0.790927 & -2.158163 \\
\hline 6 & -0.000325 & 2.398913 & 0.000000 \\
\hline 1 & -0.000171 & 2.958140 & 0.927240 \\
\hline 1 & -0.000171 & 2.958140 & -0.927240 \\
\hline
\end{tabular}

10. twisted benzyl radical 


\begin{tabular}{|c|c|c|c|}
\hline \multicolumn{4}{|c|}{$\begin{array}{l}========================================= \\
\operatorname{CAS}(4,6) / 3-21 \mathrm{G}(\mathrm{Cs}, \text { almost } \mathrm{C} 2 \mathrm{v})\end{array}$} \\
\hline & - - - - - - - - - & -----------1 & --ー-ー-ー-ー-ー-ー-ー- \\
\hline \multicolumn{4}{|c|}{ State $=2-A^{\prime} \backslash H F=-267.6509684$} \\
\hline \multicolumn{4}{|c|}{ first order saddle point } \\
\hline & $\mathrm{X}$ & $\mathrm{Y}$ & $\mathrm{Z}$ \\
\hline 6 & 0.000000 & 0.961426 & 0.000000 \\
\hline 6 & -0.000041 & -1.836082 & 0.000000 \\
\hline 6 & 1.202178 & 0.248499 & 0.000000 \\
\hline 6 & -1.202159 & 0.248524 & 0.000000 \\
\hline 6 & -1.196981 & -1.143231 & 0.000000 \\
\hline 6 & 1.196985 & -1.143234 & 0.000000 \\
\hline 1 & 2.132070 & 0.782593 & 0.000000 \\
\hline 1 & -2.132048 & 0.782622 & 0.000000 \\
\hline 1 & -2.126808 & -1.677323 & 0.000000 \\
\hline 1 & 2.126796 & -1.677355 & 0.000000 \\
\hline 1 & -0.000052 & -2.908214 & 0.000000 \\
\hline 6 & 0.000015 & 2.449182 & 0.000000 \\
\hline 1 & 0.000028 & 2.993587 & 0.923533 \\
\hline 1 & 0.000028 & 2.993587 & -0.923533 \\
\hline \multicolumn{4}{|c|}{ 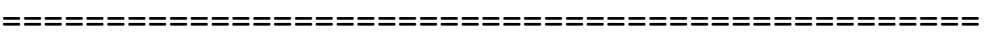 } \\
\hline \multicolumn{4}{|c|}{ B3LYP/6-31G(2df,p) (from G4 calculation) } \\
\hline \\
\hline \multicolumn{4}{|c|}{ State $=2-A \backslash E(U B 3 L Y P)=-270.911529581$} \\
\hline \multicolumn{4}{|c|}{ First order saddle point } \\
\hline \multicolumn{4}{|c|}{ G4-energy:-270.711482 (enthaply:-270.710537) } \\
\hline & $\mathrm{X}$ & $\mathrm{Y}$ & $\mathrm{Z}$ \\
\hline 6 & 0.969551 & 0.000000 & 0.000000 \\
\hline 6 & 0.249170 & -1.202832 & -0.000020 \\
\hline 6 & -1.143051 & -1.203060 & -0.000020 \\
\hline 6 & -1.844450 & 0.000000 & 0.000001 \\
\hline 6 & -1.143051 & 1.203060 & 0.000021 \\
\hline 6 & 0.249170 & 1.202832 & 0.000020 \\
\hline 1 & 0.790497 & -2.143474 & -0.000037 \\
\hline 1 & -1.679859 & -2.146331 & -0.000036 \\
\hline 1 & -2.929381 & 0.000000 & 0.000001 \\
\hline 1 & -1.679859 & 2.146331 & 0.000037 \\
\hline 1 & 0.790497 & 2.143474 & 0.000036 \\
\hline 6 & 2.444766 & 0.000000 & -0.000001 \\
\hline 1 & 3.007741 & 0.000015 & -0.927699 \\
\hline 1 & 3.007742 & -0.000016 & 0.927697 \\
\hline \multicolumn{4}{|c|}{ 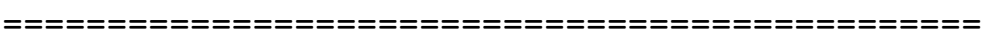 } \\
\hline \multicolumn{4}{|c|}{ B3LYP / 6-311++G** (C1) } \\
\hline \multirow{2}{*}{\multicolumn{4}{|c|}{$\begin{array}{l}\text { State }=2-\mathrm{A} \backslash \mathrm{E}(\mathrm{RB} 3 \mathrm{LYP})=-270.964950427\end{array}$}} \\
\hline & & & \\
\hline \multicolumn{4}{|c|}{ First order saddle point } \\
\hline & $\mathrm{X}$ & $\mathrm{Y}$ & $\mathrm{Z}$ \\
\hline 6 & 0.970274 & -0.000001 & 0.000003 \\
\hline 6 & 0.249757 & -1.204126 & -0.000009 \\
\hline 6 & -1.143903 & -1.203840 & -0.000008 \\
\hline 6 & -1.845923 & 0.000001 & 0.000004 \\
\hline 6 & -1.143901 & 1.203841 & 0.000007 \\
\hline 6 & 0.249760 & 1.204125 & 0.000002 \\
\hline 1 & 0.789272 & -2.144937 & -0.000021 \\
\hline 1 & -1.680559 & -2.146314 & -0.000015 \\
\hline 1 & -2.930094 & 0.000002 & 0.000007 \\
\hline 1 & -1.680556 & 2.146316 & 0.000014 \\
\hline 1 & 0.789276 & 2.144935 & 0.000003 \\
\hline 6 & 2.447287 & -0.000001 & 0.000003 \\
\hline 1 & 3.006274 & 0.000160 & -0.929152 \\
\hline 1 & 3.006275 & -0.000158 & 0.929158 \\
\hline
\end{tabular}


11. planar benzyl anion

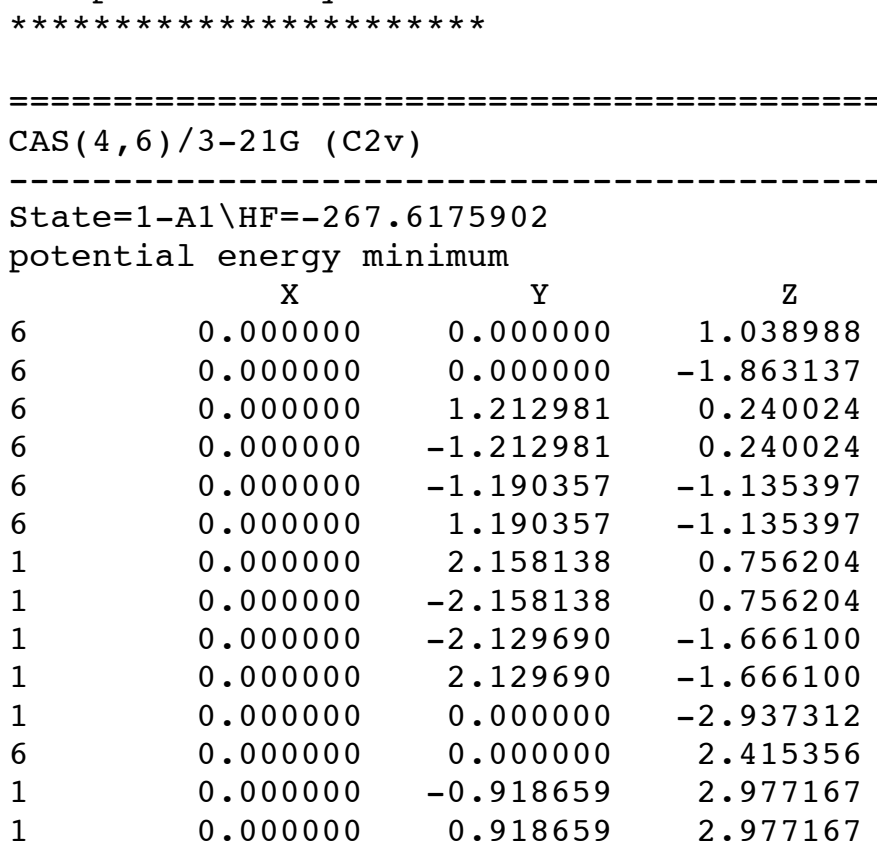

\begin{tabular}{|c|c|c|c|}
\hline \multicolumn{4}{|c|}{ UB3LYP/6-31G(2df,p) (Cs) (from G4 calculation) } \\
\hline \multicolumn{4}{|c|}{$\begin{array}{l}\text { State }=1-A^{\prime} \backslash E(R B 3 L Y P)=-270.941745773 \\
\text { potential energy minimum } \\
G 4-e n e r g y:-270.763479 \text { (enthaply:-270.762535) }\end{array}$} \\
\hline & $\mathrm{X}$ & $\mathrm{Y}$ & $\mathrm{Z}$ \\
\hline 6 & 0.000027 & 1.043226 & 0.000000 \\
\hline 6 & 0.000020 & 0.242331 & 1.209670 \\
\hline 6 & 0.000020 & -1.137360 & 1.195446 \\
\hline 6 & -0.000038 & -1.877630 & 0.000000 \\
\hline 6 & 0.000020 & -1.137360 & -1.195446 \\
\hline 6 & 0.000020 & 0.242331 & -1.209670 \\
\hline 1 & -0.000005 & 0.766425 & 2.165293 \\
\hline 1 & 0.000011 & -1.667326 & 2.150236 \\
\hline 1 & 0.000216 & -2.963230 & 0.000000 \\
\hline 1 & 0.000011 & -1.667326 & -2.150236 \\
\hline 1 & -0.000005 & 0.766425 & -2.165293 \\
\hline 6 & -0.000077 & 2.422733 & 0.000000 \\
\hline 1 & -0.000087 & 2.987704 & 0.927716 \\
\hline 1 & -0.000087 & 2.987704 & -0.927716 \\
\hline \multirow{2}{*}{\multicolumn{4}{|c|}{$\begin{array}{l}============================================== \\
\text { B3LYP } / 6-311++G * \text { (CS ) }\end{array}$}} \\
\hline & & & \\
\hline \multicolumn{4}{|c|}{$\begin{array}{l}\text { State }=1-A^{\prime} \backslash \mathrm{E}(\mathrm{RB} 3 \mathrm{LYP})=-271.017953503 \\
\text { Potential energy minimum }\end{array}$} \\
\hline & $\mathrm{x}$ & $\mathrm{Y}$ & $\mathrm{z}$ \\
\hline 6 & 0.000062 & 1.037380 & 0.000000 \\
\hline 6 & 0.000021 & 0.243445 & 1.211190 \\
\hline 6 & 0.000021 & -1.138740 & 1.199188 \\
\hline 6 & -0.000147 & -1.877672 & 0.000000 \\
\hline 6 & 0.000021 & -1.138740 & -1.199188 \\
\hline 6 & 0.000021 & 0.243445 & -1.211190 \\
\hline 1 & 0.000027 & 0.767424 & 2.164999 \\
\hline 1 & -0.000030 & -1.668948 & 2.151395 \\
\hline 1 & 0.000490 & -2.962413 & 0.000000 \\
\hline 1 & -0.000030 & -1.668948 & -2.151395 \\
\hline
\end{tabular}




$\begin{array}{lrrr}1 & 0.000027 & 0.767424 & -2.164999 \\ 6 & -0.000067 & 2.428048 & 0.000000 \\ 1 & -0.000037 & 2.991231 & 0.927787 \\ 1 & -0.000037 & 2.991231 & -0.927787\end{array}$

12. twisted benzyl anion

$* * * * * * * * * * * * * * * * * * * * * * * *$

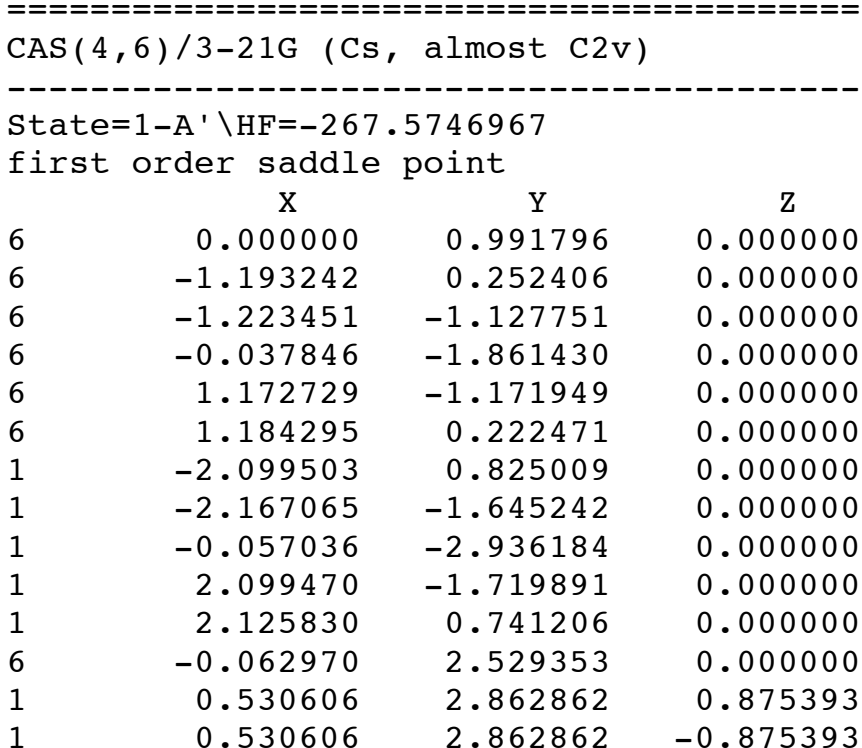

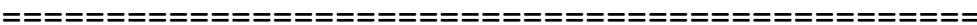
B3LYP/6-31G(2df,p) (from G4 calculation)

State $=1-A \backslash E(R B 3 L Y P)=-270.895466362$

first order saddle point

G4-energy:-270.726427 (enthaply:-270.725483)

$$
\mathrm{X}
$$

$$
\mathrm{Y}
$$

$$
\mathrm{Z}
$$

$\begin{array}{rrrr}6 & -1.011736 & -0.045063 & 0.000052 \\ 6 & -0.207229 & -1.207442 & 0.000028 \\ 6 & 1.187371 & -1.175788 & -0.000044 \\ 6 & 1.866310 & 0.042347 & -0.000096 \\ 6 & 1.116273 & 1.220351 & -0.000077 \\ 6 & -0.276220 & 1.168252 & -0.000007 \\ 1 & -0.744243 & -2.153194 & 0.000074 \\ 1 & 1.751756 & -2.107950 & -0.000057 \\ 1 & 2.953451 & 0.075844 & -0.000149 \\ 1 & 1.626260 & 2.184216 & -0.000115 \\ 1 & -0.842293 & 2.098693 & 0.000007 \\ 6 & -2.514174 & -0.172599 & 0.000143 \\ 1 & -2.854204 & 0.461075 & 0.857393 \\ 1 & -2.854300 & 0.460961 & -0.857151\end{array}$

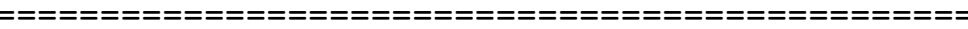
B3LYP / 6-311++G** (C1)

State $=1-A \backslash E(R B 3 L Y P)=-270.977388113$

First order saddle point

$\mathrm{X}$

1.006956

0.208790

$-1.187754$

$-1.867851$

$-1.119663$
$\mathrm{Y}$

$-0.044352$

$-1.210484$

$-1.177138$

0.041582

1. 221293
Z

0.000536

0.000270

$-0.000100$

$-0.000222$

0.000039 


$\begin{array}{rrrr}6 & 0.275526 & 1.171760 & 0.000281 \\ 1 & 0.739997 & -2.157108 & 0.000221 \\ 1 & -1.749040 & -2.109066 & -0.000352 \\ 1 & -2.953921 & 0.073285 & -0.000501 \\ 1 & -1.627929 & 2.184075 & -0.000163 \\ 1 & 0.841822 & 2.099161 & 0.000067 \\ 6 & 2.505897 & -0.144993 & -0.000461 \\ 1 & 2.908135 & 0.382227 & -0.883407 \\ 1 & 2.909527 & 0.381424 & 0.882082\end{array}$

13. Methane

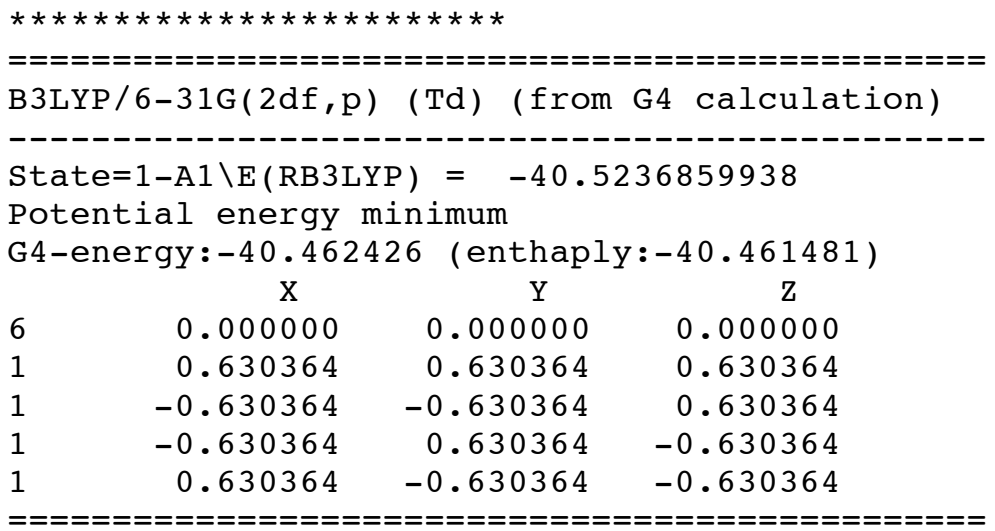

14. Methyl cation

$* * * * * * * * * * * * * * * * * * * * * * * *$

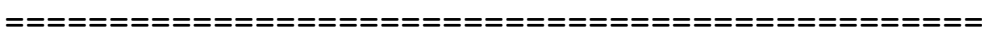
B3LYP/6-31G(2df,p) (D3h) (from G4 calculation)

State $=1-A 1^{\prime} \backslash E($ RB3LYP $)=-39.4848566083$

Potential energy minimum

G4-energy:-39.434823 (enthaply:-39.433879)

\begin{tabular}{|c|c|c|c|}
\hline & $X$ & $Y$ & Z \\
\hline 6 & 0.000000 & 0.000000 & 0.000000 \\
\hline & 0.000000 & 1.095321 & 0.000000 \\
\hline & -0.948576 & -0.547661 & 0.000000 \\
\hline & 0.948576 & -0.547661 & 0.000000 \\
\hline
\end{tabular}

15. Methyl radical

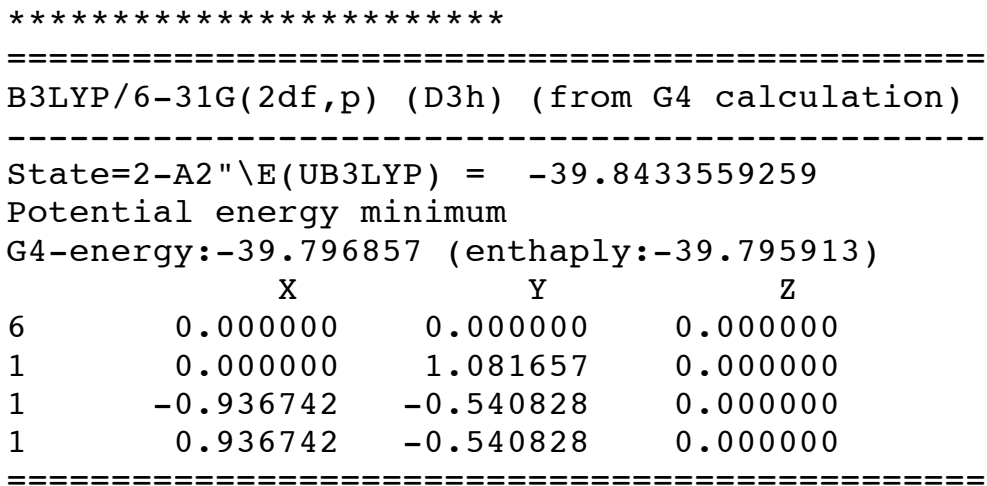

16. Methyl anion

$* * * * * * * * * * * * * * * * * * * * * * * *$

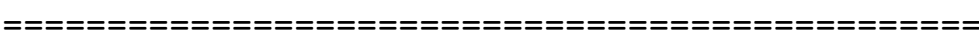
B3LYP/6-31G(2df,p) (D3h) (from G4 calculation)

State $=1-A 1 \backslash E($ RB3LYP $)=-39.8000335451$

Potential energy minimum 
G4-energy:-39.796576 (enthaply:-39.795632)

$\mathrm{X}$

0.000000

0.000000

$-0.860580$

0.860580

0.000000

0.993712

$-0.496856$

$-0.496856$
0.180122

$-0.360244$

$-0.360244$

$-0.360244$ 